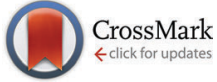

Cite this: Phys. Chem. Chem. Phys., 2016, 18, 28325

Received 24th August 2016, Accepted 9th September 2016

DOI: $10.1039 / c 6 c p 05849 c$

www.rsc.org/pccp

\section{Application of classical simulations for the computation of vibrational properties of free molecules $\dagger$}

\author{
Denis S. Tikhonov, ${ }^{\text {ab }}$ Dmitry I. Sharapa, ${ }^{c}$ Jan Schwabedissen $^{a}$ and \\ Vladimir V. Rybkin*d
}

\begin{abstract}
In this study, we investigate the ability of classical molecular dynamics (MD) and Monte-Carlo (MC) simulations for modeling the intramolecular vibrational motion. These simulations were used to compute thermally-averaged geometrical structures and infrared vibrational intensities for a benchmark set previously studied by gas electron diffraction (GED): $\mathrm{CS}_{2}$, benzene, chloromethylthiocyanate, pyrazinamide and 9,12- $\mathrm{I}_{2}$ 1,2-closo- $\mathrm{C}_{2} \mathrm{~B}_{10} \mathrm{H}_{10}$. The MD sampling of NVT ensembles was performed using chains of Nose-Hoover thermostats $(\mathrm{NH})$ as well as the generalized Langevin equation thermostat (GLE). The performance of the theoretical models based on the classical MD and MC simulations was compared with the experimental data and also with the alternative computational techniques: a conventional approach based on the Taylor expansion of potential energy surface, path-integral MD and MD with quantum-thermal bath (QTB) based on the generalized Langevin equation (GLE). A straightforward application of the classical simulations resulted, as expected, in poor accuracy of the calculated observables due to the complete neglect of quantum effects. However, the introduction of a posteriori quantum corrections significantly improved the situation. The application of these corrections for MD simulations of the systems with large-amplitude motions was demonstrated for chloromethylthiocyanate. The comparison of the theoretical vibrational spectra has revealed that the GLE thermostat used in this work is not applicable for this purpose. On the other hand, the $\mathrm{NH}$ chains yielded reasonably good results.
\end{abstract}

\section{Introduction}

\subsection{Geometrical and vibrational studies of free molecules}

The studies of structural and vibrational properties of free molecules in the ground state have been around in the scientific community since the dawn of quantum chemistry and modern physical methods. ${ }^{1-4}$ The value of such data is not exhausted by the information relevant for a specific chemical application.

\footnotetext{
${ }^{a}$ Universität Bielefeld, Lehrstuhl für Anorganische Chemie und Strukturchemie, Universitätsstrasse 25, 33615, Bielefeld, Germany.

E-mail: denis.tikhonov@uni-bielefeld.de

${ }^{b}$ M. V. Lomonosov Moscow State University, Department of Physical Chemistry, GSP-1, 1-3 Leninskiye Gory, 119991 Moscow, Russia

${ }^{c}$ Computer-Chemie-Centrum and Interdisciplinary Center for Molecular Materials, Department Chemie und Pharmazie, Friedrich-Alexander-Universität Erlangen-Nürnberg, 91052 Erlangen, Germany

${ }^{d}$ ETH Zurich, Department of Materials, Wolfgang-Pauli-Strasse 27, CH-8093 Zurich, Switzerland. E-mail: vladimir.rybkin@mat.ethz.ch

$\dagger$ Electronic supplementary information (ESI) available: UNEX input files that were used to perform the comparison of theoretical GED models with experimental data for BNZ, CMTC, PZA and ICB can be found. These files contain computed $r_{\mathrm{e}}$ geometries and vibrational parameters $\left(l, r_{\mathrm{e}}-r_{\mathrm{a}}\right.$ and $\left.6 \cdot \kappa\right)$ as well as experimental $s M(s)$ curves. See DOI: $10.1039 / \mathrm{c} 6 \mathrm{cp} 05849 \mathrm{c}$
}

They have been used for many purposes in theoretical chemistry. ${ }^{5,6}$ Notable examples are:

- development of valence theory ${ }^{5,7}$ by Linus Pauling,

- theoretical background and experimental support for the valence shell electron pair repulsion theory (VSPER), ${ }^{6}$

- usage of experimental geometrical parameters of molecules in the gas phase for parameterization of AM1 and MNDO semiempirical models, ${ }^{8-10}$ and the functional MN15-L. ${ }^{11}$

Therefore, the experimental gas-phase molecular structure studies should not be underestimated. The three main methods for investigation of the ground state geometrical or/and vibrational properties of free molecules are:

- gas-phase electron diffraction (GED), ${ }^{7,12}$

- IR or Raman vibrational spectroscopy (VSp), ${ }^{4,13}$

- rotational spectroscopy (RSp). ${ }^{4,13}$

The interpretation can be done by solving either direct or inverse problems. ${ }^{14,15}$ In both cases a theoretical model is required. A direct problem is basically a simple comparison of experimental data with that computed from the model. In this case, a theoretical model is usually based on quantum chemical calculations (QC). The inverse problem supposes that a theoretical model can be parametrized with a set of variables 
(e.g. geometrical parameters, ${ }^{16-24}$ the parametric form of potential energy for internal motions ${ }^{18,21,25}$ or some mean parameters such as scale factors for harmonic force fields $\left.{ }^{18,20,21}\right)$. These experimental parameters are obtained by fitting the theoretical model to the experimental data. Unfortunately, all the parameters of the model can be unequivocally obtained from the experimental data only for small molecules, otherwise the inverse problem becomes ill-defined. ${ }^{26}$ Therefore, in most cases some parameters should be taken as they are from the theoretical computations based mainly on QC. Consequently, the accuracy of these parameters is crucial. Fortunately, the effects arising from the choice of the QC approximation are significantly smaller than the experimental uncertainties and systematic errors of the theoretical models used for fitting. As a consequence, computationally cheap QC approaches can be used in particular to account for molecular vibrations without any loss of accuracy. ${ }^{27}$

\subsection{Theoretical models for intramolecular vibrations}

Let us assume the applicability of the Born-Oppenheimer (B-O) approximation. The direct solution of Schrödinger equation for rovibrational levels on the preconstructed potential energy surface (PES) is possible only for small systems consisting of a few atoms. ${ }^{28-30}$ Therefore, for larger systems simplifications are introduced. In cases of semi-rigid molecules, it is the separation of the rotational degrees of freedom, which are then treated as a rigid rotor. A further simplification is the use of the harmonic oscillator approximation for the vibrations, giving rise to the rigid rotor-harmonic oscillator (RR-HO) approximation. ${ }^{13,31,32}$ This model is a very reasonable zeroth approximation for the molecules with small-amplitude motion. All the leftover effects, i.e. vibration-rotation interaction and the higher-order (anharmonic) terms in Taylor expansion of PES can be introduced via perturbation theory. ${ }^{4,15,19,33}$ In cases of non-rigid molecules the technique described above can be augmented by the direct treatment of the large-amplitude motion (LAM), for example, as it is described in ref. 34. This model gives the observables for GED, VSp and $\mathrm{RSp}$ and also provides a framework for analysis and discussion of the experiments.

The anharmonic effects themselves are very important for the successful interpretation of the experimental data. ${ }^{20,35-37}$ The cubic force field is required for all the models (i.e. GED, VSp and RSp). The quartic force field $\left(\frac{\partial^{4} V}{\partial q_{i} \partial q_{j} \partial q_{k} \partial q_{l}}\right)$ is not that necessary for GED and RSp since the main aharmonic shift of the atoms from the equilibrium position appears mainly due to the cubic force field. ${ }^{19,38}$ In the case of VSp, the quartic force field appears in the first order of perturbation theory only as semidiagonal elements $\left(\frac{\partial^{4} V}{\partial Q_{i}^{2} \partial Q_{j}^{2}}\right)$ in normal coordinates $Q .{ }^{38}$ Thus, its computation takes the same order of time as the computation of the harmonic force field. In this work, we do not consider complete quartic force fields.

The higher the order of the PES Taylor expansion term, the more computationally expensive it is. The number of cubic force constants grows as $O\left(N^{3}\right)$ with the size of the system, the cost of each constant growth being dependent on the electronic

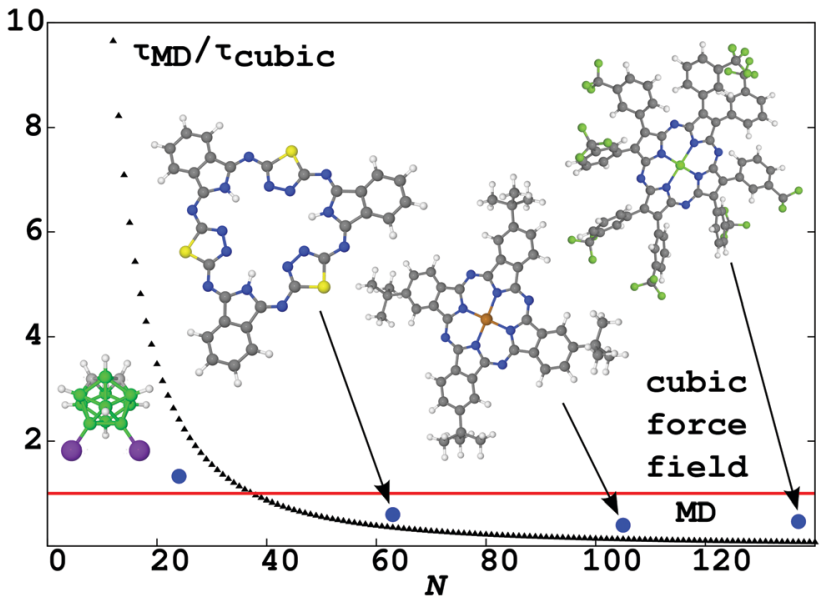

Fig. 1 Relative cost of MD simulation for the computation of the cubic force field as approximated by eqn (1), $a=10$ and $b=5$. Triangles show the theoretical behavior at $\nu_{\max } / \nu_{\min }=1000$, circles demonstrate the application of this relation for some molecules that were recently investigated using GED. ${ }^{23,43-45}$

structure method and its implementation. Consequently, at one point it becomes cheaper to use alternative techniques to determine the gas-phase structure, for example, classical $a b$ initio molecular dynamics (MD) or Monte-Carlo (MC) simulations. ${ }^{39-41}$ An estimate for the relation of times for performing MD simulations $\tau_{\mathrm{MD}}$ to the computation of the cubic force field $\tau_{\mathrm{CFF}}$ can be made as follows: $:^{23,42}$

$$
\frac{\tau_{\mathrm{MD}}}{\tau_{\mathrm{CFF}}}=\frac{a \cdot b \cdot\left(\nu_{\max } / \nu_{\min }\right)}{36 N^{2}},
$$

where $\nu_{\max }$ and $\nu_{\min }$ are the highest and the lowest vibrational frequencies for the chosen molecule, $a$ shows how many time steps are required to cover the period of the fastest vibration and $b$ shows how many periods of the lowest vibration are in the total length of the trajectory. Thus, $a$ and $b$ control the quality of the MD simulation. An illustration of this relation can be found in Fig. 1.

In cases of non-rigid molecules with multiple LAMs a multidimensional PES scan along the LAM coordinates is required in addition to a cubic force field. Therefore for such systems the computational cost of the conventional model grows even faster.

\subsection{Application of classical simulations for gas electron diffraction and vibrational spectroscopy}

Classical ab initio MD simulations have been used to simulate VSp for quite a long time. ${ }^{46}$ The observable in a VSp experiment is the intensity $(I) v s$. the frequency $(\nu)$. In the case of infrared absorption, the theoretical spectrum from MD is calculated as follows: ${ }^{47-49}$

$$
I(\nu) \propto \frac{1}{n(\nu)} \underbrace{\nu \tanh \left(\frac{h \nu}{2 k_{\mathrm{B}} T}\right)}_{Q} \operatorname{Re} \int_{0}^{\infty} \mathrm{e}^{-i 2 \pi \nu t}\langle\mathbf{d}(t), \mathbf{d}(0)\rangle \mathrm{d} t,
$$

where $\mathbf{d}$ is the electronic dipole moment of the system, $\langle\mathbf{d}(t)$, $\mathbf{d}(0)\rangle=f(t)$ is the autocorrelation function of the dipole moment, 
and $n(\nu)$ is the refractive index. For the gas phase, $n \approx 1 .^{50}$ There also exist several other possibilities to introduce the quantum correction $(Q)$ to the intensities. ${ }^{50,51}$

The GED observable is the reduced molecular scattering intensity $(s M(s))$. In the case of semi-rigid molecules it is given by the equation: ${ }^{12,52}$

$$
s M(s)=\sum_{i=1}^{N} \sum_{j=1}^{j<i} \frac{2 g_{i j}(s)}{r_{a, i j}} \exp \left(-\frac{l_{i j}^{2} s^{2}}{2}\right) \sin \left(s r_{a, i j}-s^{3} \kappa_{i j}\right),
$$

where $s=\frac{4 \pi}{\lambda} \sin \left(\frac{\theta}{2}\right)$ is the scattering coordinate, $\lambda$ is the wavelength of the electrons, $\theta$ is the scattering angle, $i$ and $j$ are numbers of atoms, $N$ is the total number of atoms, and $g_{i j}$ is the function characterizing the scattering ability of the pair of atoms $i$ and $j$. The three parameters left, i.e. average distance $r_{\mathrm{a}}$, mean vibrational amplitude $l$ and asymmetry parameter $\kappa$, are the moments of probability distribution function $P(r) / r$, where $r$ is the distance, $P(r)$ is the probability distribution for the chosen pair of atoms. They are expressed by the following equations: ${ }^{15,52,53}$

$$
\begin{gathered}
r_{\mathrm{g}}=\langle r\rangle, \\
r_{\mathrm{a}}=\left\langle\frac{1}{r}\right\rangle^{-1} \approx r_{\mathrm{g}}-\frac{l^{2}}{r_{\mathrm{e}}}, \\
l^{2}=\left\langle r^{2}\right\rangle-\langle r\rangle^{2}, \\
\kappa=\frac{1}{6}\left(\left\langle r^{3}\right\rangle-3\langle r\rangle\left\langle r^{2}\right\rangle+2\langle r\rangle^{3}\right),
\end{gathered}
$$

where brackets \langle\rangle denote averaging over vibrations at the temperature of the experiment and $r_{\mathrm{e}}$ denotes the equilibrium distance between the atoms that is found at the minimum of the PES. Parameters $l, \kappa$ and $\left(r_{\mathrm{e}}-r_{\mathrm{a}}\right)$ describe the internal vibrations in the semi-rigid molecules to a high degree of accuracy. Therefore if we know these parameters we can find the experimental equilibrium structure that can be then compared to that found by the QC computations and RSp.

\subsection{A brief description of the current work}

This article is a continuation of a recently published work. ${ }^{54}$ In the current paper, we thoroughly test the performance of the theoretical methods for 5 previously investigated molecules:

(1) $\mathrm{CS}_{2},{ }^{16}$

(2) benzene (BNZ), ${ }^{54}$

(3) chloromethylthiocyanate (CMTC), ${ }^{22}$

(4) pyrazinamide (PZA), ${ }^{24}$

(5) 9,12-I ${ }_{2}-1,2$-closo-C2B10H10 (ICB). ${ }^{23}$

All of them were investigated using GED. Different theoretical thermally-averaged structures for these molecules including those obtained on the basis of classical MD and MC simulations were compared with the experimental data. During the tests the importance of quantum effects as well as the performance of different $N V T$ ensemble sampling techniques was investigated. The performance of different MD thermostats was also investigated for the computation of VSp.

\section{Computational and experimental details}

\subsection{Theoretical equilibrium structures}

Equilibrium geometries of the $\mathrm{CS}_{2}$ molecule at $\operatorname{CCSD}(\mathrm{T}), \mathrm{MP} 2$, PBE0, B3LYP and BP86 levels of theory with cc-pVnZ, cc-pCVnZ $(n=2, \ldots 6)$ and cc-pwCVnZ $(n=2, \ldots 5)$ basis sets were obtained using Gaussian $09^{55}$ software. Additional optimizations at DCFT (OCD-12) with cc-pVn $(n=2, \ldots 4)$ basis sets and at CCSDT/cc-pVTZ levels of theory were done using Psi $4^{56}$ and CFour ${ }^{57}$ packages, respectively.

Optimizations of structures of BNZ, CMTC and PZA at MP2, PBE0, B3LYP and BP86 levels of theory with the cc-pVTZ basis set were done using Firefly 8 program. ${ }^{58}$ Computation of equilibrium geometry of ICB at BP86/SDB-cc-pVTZ and PBE0/SDB-ccpVTZ was performed using Gaussian $09 .{ }^{55}$ Equilibrium geometries of BNZ and CMTC at CCSD(T)/cc-pVTZ level of theory and ICBb at MP2/SDB-cc-pVTZ and B3LYP/SDB-cc-pVTZ levels were taken from the results of the earlier studies. ${ }^{22,23,59}$

CCSDT, CCSD(T), ${ }^{60} \mathrm{MP} 2,{ }^{61}$ DCFT/OCD-12, ${ }^{62}$ PBE0,${ }^{63}$ B3LYP,${ }^{64-66}$ BP86 ${ }^{67-69}$ methods were used as implemented in software mentioned. Basis sets cc-pVnz, ${ }^{70}$ cc-pCVnz, ${ }^{71}$ cc-pwCVnz ${ }^{72}$ and SDB-cc-pVTZ (with Stuttgart-Dresden-Bonn relativistic pseudopotentials ${ }^{73}$ were taken from EMSL BSE Library. ${ }^{74,75}$

\subsection{Simulation of intramolecular atomic vibrations}

2.2.1 Vibrational parameters for GED. In this work, different types of models were used for the computation of GED vibrational parameters $\left(l, r_{\mathrm{e}}-r_{\mathrm{a}}\right.$, and $\left.\kappa\right)$. The first group of methods is conventional for GED. The RR-HO approximation is taken as the zeroth-order approximation, whereas the effects of anharmonicity and rotation-vibration interactions are introduced by the perturbation theory. These calculations employ quadratic and cubic force fields.

- ElDiff - the parameters were computed using the ElDiff program. $^{15}$

- VM - the parameters were computed using the VibModule program. ${ }^{76}$ It is a new implementation of Sipachev's Shrink software. ${ }^{77-80}$

The second group consists of classical methods: MD and MC simulations.

- NH, GLE - the parameters were computed directly from the MD trajectory. NH denotes that the NVT ensemble was simulated using the Nose-Hoover chain thermostat, ${ }^{81-83}$ while GLE stands for the colored-noise generalized Langevin equation thermostat. ${ }^{84}$ The values of vibrational parameters are obtained directly from eqn (4)-(7), as described previously. ${ }^{52}$

- MC - the parameters were computed directly from the classical Metropolis Monte-Carlo simulation. ${ }^{85}$

The third group comprises classical simulations a posteriori corrected for the quantum effects. The corrections are implemented in the Qassandra program (QA). ${ }^{42}$ They are denoted as $\mathrm{NH}+\mathrm{QA}, \mathrm{GLE}+\mathrm{QA}$ and $\mathrm{MC}+\mathrm{QA}$, where the first name corresponds to the initial classical simulation given in the second group. Finally, the last group of methods includes quantum molecular dynamics simulations: path-integral MD 
Table 1 Computational details about vibrational models

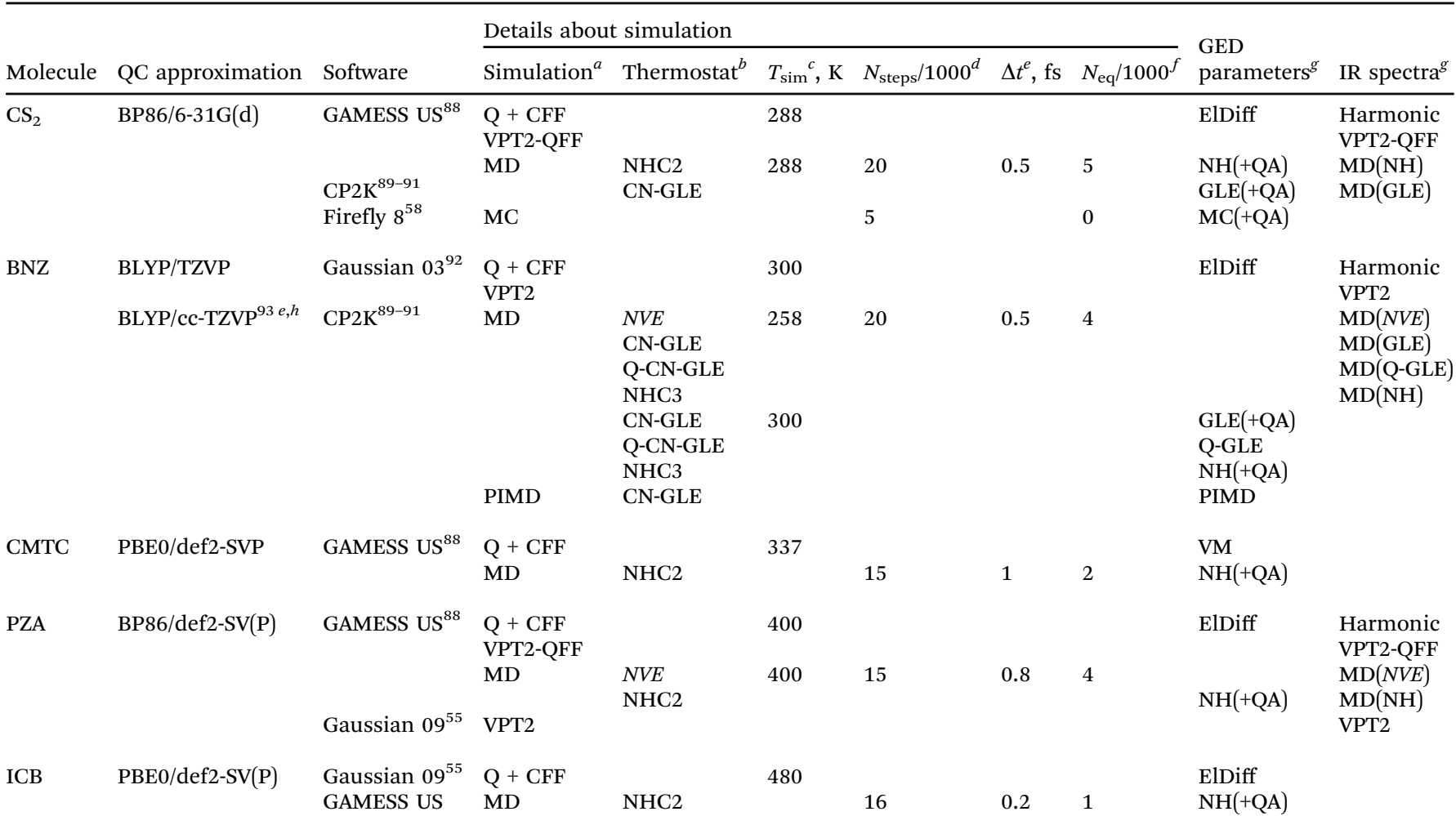

${ }^{a}$ The following notations were used: Q + CFF is quadratic and cubic force field computations;VPT2-QFF and VPT2 denote 2nd order vibrational perturbation theory based on cubic and quartic force fields, as implemented in GAMESS US ${ }^{94}$ and Gaussian ${ }^{38}$, respectively; MD is molecular dynamics; PIMD is path-integral molecular dynamics; MC is Metropolis Monte-Carlo simulation. ${ }^{b}$ The thermostats used are denoted as the following: $N V E$ is constant energy MD. NHC $n$ is the chain of $n$ Nose-Hoover thermostats. (Q-)CN-GLE are quantum and canonical colored-noise generalized Langevin equation thermostats. ${ }^{c} \mathrm{~T}$ is the temperature of simulation that corresponds to the experimental temperatures in ref. Morino and Iijima, ${ }^{16}$ Tikhonov et al.,${ }^{54}$ Berrueta Martinez et al.,${ }^{22}$ Vishnevskiy et al. ${ }^{23}$ Tikhonov et $a ._{.}{ }^{24}$ and in the current work. ${ }^{d} N_{\text {steps }}$ is the number of steps performed in MD or MC simulation. ${ }^{e}$ Time step of MD simulations. ${ }^{f} N_{\text {steps }}$ is the number of steps skipped in the begining of MD or MC trajectory to account for the equilibration phase. ${ }^{g}$ These columns give the notations of the models used further in the text. ${ }^{h}$ The GoedeckerTeter-Hutter pseudopotentials have been used, ${ }^{95}$ electron repulsion integrals were calculated using the Gaussian-and-Plane-Waves method ${ }^{96}$ with a non-periodic Poisson solver using the auxiliary plane-wave basis with a 400 Ry cutoff, the SCF guess was propagated using the always stable predictor-corrector method. ${ }^{97}$

(PIMD) and the GLE dynamics using the quantum thermostat (Q-GLE). ${ }^{86,87}$ PIMD simulations were equilibrated with the GLE thermostat $^{84,87}$ The parameters were taken directly from the trajectory.

The details of the computation of vibrational models are given in Table 1.

\subsection{Infrared vibrational spectra}

MD trajectories were converted into spectra using in-house AWK scripts. A Fourier transformation of the dipole autocorrelation function (eqn (2)) was performed with the help of the Gaussian window function $w(t)=\exp \left(-t^{2} / \tau^{2}\right)$. The $\tau$ for $\mathrm{CS}_{2}$, BNZ and PZA were 1 ps. Since time steps in the corresponding simulations are quite large, a correction ${ }^{98}$ for the finite-time step frequency shifts ${ }^{99}$ was applied.

Harmonic and VPT2 spectra of both molecules were converted into frequency $v s$. intensity spectra using the Lorenzian profile function with a FHWH of $10 \mathrm{~cm}^{-1}$. For harmonic and VPT2 spectra of PZA and harmonic spectra of BNZ Gabedit software was used. ${ }^{100}$ VPT2 spectra of BNZ was converted into a curve manually using computed anharmonic fundamentals and intensities from the harmonic approximation.

\subsection{Comparison with GED data}

The construction of theoretical GED models for BNZ, CMTC, PZA and ICB, their comparison with the experimental data and computation of radial distribution curves were done using UNEX software. ${ }^{101}$ Theoretical $s M(s)$ were scaled to give the best fit to their experimental analogs using the least-squares method:

$$
\sum_{k}\left(a \cdot s_{k} M_{\text {theor }}\left(s_{k}\right)-s_{k} M_{\exp }\left(s_{k}\right)\right)^{2} \rightarrow \min ,
$$

where $a$ is the refined scaling factor. Experimental GED values $r_{\mathrm{g}}$ and $l$ for $\mathrm{CS}_{2}$ were taken from ref. Morino and Iijima. ${ }^{16}$ Experimental $s M(s)$ curves for CMTC, PZA and ICBb were taken from ref. Berrueta Martinez et al., ${ }^{22}$ Tikhonov et al. ${ }^{24}$ and Vishnevskiy et $a l^{23}$ Experimental data for BNZ at $300 \mathrm{~K}$ were obtained in this work. The description of the experiment is given in the next paragraph. 
Table 2 Conditions of the GED experiment for BNZ

\begin{tabular}{llll}
\hline Parameter & & MD & LD \\
\hline$d_{\text {nozzle-film }}$ & {$[\mathrm{mm}]$} & 250.0 & 500.0 \\
$U_{\text {acceleration }}$ & {$[\mathrm{kV}]$} & 60 & 60 \\
$I_{\text {fast electrons }}$ & {$[\mu \mathrm{A}]$} & 1.53 & 1.52 \\
$\lambda_{\text {electrons }}$ & {$[\AA]$} & 0.048771 & 0.048678 \\
$T_{\text {nozzle }}$ & {$[\mathrm{K}]$} & 299 & 298 \\
$p_{\text {sample }}$ & {$[\mathrm{Pa}]$} & $2.3 \times 10^{-4}$ & $1.3 \times 10^{-4}$ \\
$p_{\text {residual gas }}$ & {$[\mathrm{Pa}]$} & $8.1 \times 10^{-6}$ & $1.2 \times 10^{-5}$ \\
$t_{\text {exposure }}$ & {$[\mathrm{s}]$} & 6 & 7 \\
$s_{\text {range }}$ & {$\left[\AA^{-1}\right]$} & $6.2-32.2$ & $2.2-16.4$ \\
& & &
\end{tabular}

2.4.1 GED experiment for BNZ. A sample of BNZ with purity $\geq 99.5 \%$ was purchased from Roth-Chemie GmbH and used with no further purification.

The electron diffraction patterns were recorded on the recently improved Balzers Eldigraph KDG2 gas electron diffractometer. ${ }^{102,103}$ Data were collected at two different nozzle-to-detector distances, namely 250.0 (MD) and $500.0 \mathrm{~mm}$ (LD). The electron diffraction patterns were measured on Fuji BAS IP MP 2025 imaging plates, which were scanned using a calibrated Fuji BAS 1800II scanner. The temperature of the experiment was maintained as close to $300 \mathrm{~K}$ as possible in order to be applicable for multiple computations done in the previous study. ${ }^{54}$

The intensity curves were obtained from scanned images by applying the method described in detail elsewhere. ${ }^{104}$ Electron wavelengths were refined ${ }^{105}$ using diffraction patterns of $\mathrm{CCl}_{4}$, recorded along with the substances under investigation. Background was extracted from total intensity curves using the UNEX program. ${ }^{101}$

The summary of experimental conditions is given in Table 2.

\section{Results and discussion}

\section{1 $\mathrm{CS}_{2}$ : the simplest example}

3.1.1 Computation of the $\mathbf{C}-\mathbf{S}$ bond length. In the case of $\mathrm{CS}_{2}$, the experimental $r_{\mathrm{g}}(\mathrm{C}-\mathrm{S})=1.559(2) \AA$ at $288 \mathrm{~K}$ is known. ${ }^{16}$ This gives a natural limit for the computed equilibrium bond length $r_{\mathrm{e}}(\mathrm{C}-\mathrm{S})<r_{\mathrm{g}}(\mathrm{C}-\mathrm{S})($ at $\forall \mathrm{T})$. This is due to the existence of an interatomic repulsion wall: the smaller distances are significantly less favored than the larger ones in course of the vibrational motion. Fig. 2 and 3 (exact numbers listed in Table 4) show that the equilibrium bond length depends significantly on the used basis set. For all used methods it is significantly overestimated when small basis sets are used (in comparison to the bigger basis sets at the same level of theory). $\operatorname{CCSD}(\mathrm{T})$ calculations with different sub-families of Dunning basis sets converge to the same bond length, $1.556 \AA$, that is used by us in the following discussion. While Halkier et al. ${ }^{106}$ found very small difference between bond lengths obtained using $\operatorname{CCSD}(\mathrm{T})$ and $\mathrm{CCSDT}$, in the case of $\mathrm{CS}_{2}$ this difference reaches $0.5 \mathrm{pm}$ on the cc-pVTZ basis set. However Fig. 2 shows a general agreement between CCSDT and $\operatorname{CCSD}(\mathrm{T})$ results. MP2 shows the same convergence as $\operatorname{CCSD}(\mathrm{T})$ but predicts a somewhat shorter equilibrium bond length (1.554 ̊). The three compared DFT-functionals show different behavior in convergence and, moreover, converge to different

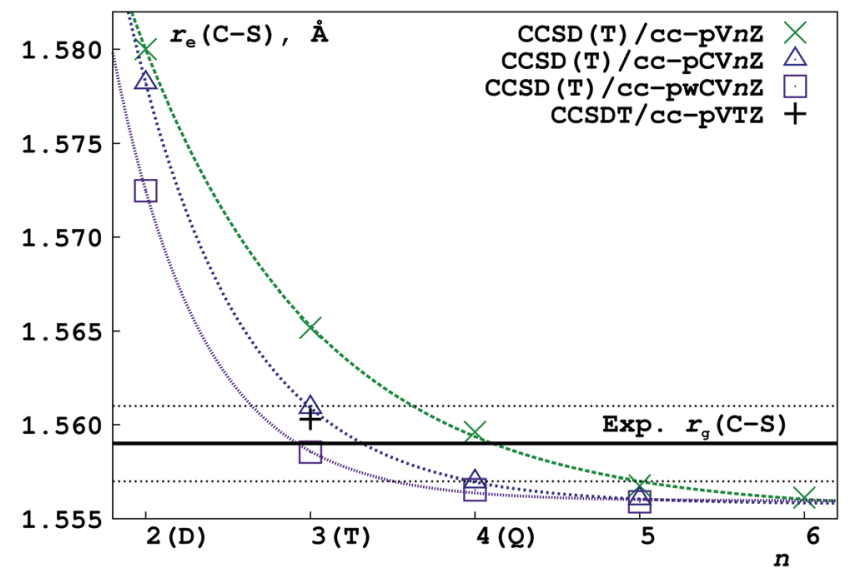

Fig. $2 r_{\mathrm{e}}(\mathrm{C}-\mathrm{S})$ in $\mathrm{CS}_{2}$ at $\mathrm{CCSD}(\mathrm{T})$ and CCSDT levels of theory with $\mathrm{cc}-\mathrm{pV} n \mathrm{n}, \mathrm{cc}-\mathrm{pCVnZ}(n=2, \ldots, 6)$ and cc-pwCVnZ $(n=2, \ldots, 5)$ basis sets. Solid horizontal line represents the experimental $r_{\mathrm{g}}(\mathrm{C}-\mathrm{S})$ bond length $\mathrm{a} 288 \mathrm{~K}$ and dotted horizontal lines represent its $3 \sigma .{ }^{16}$

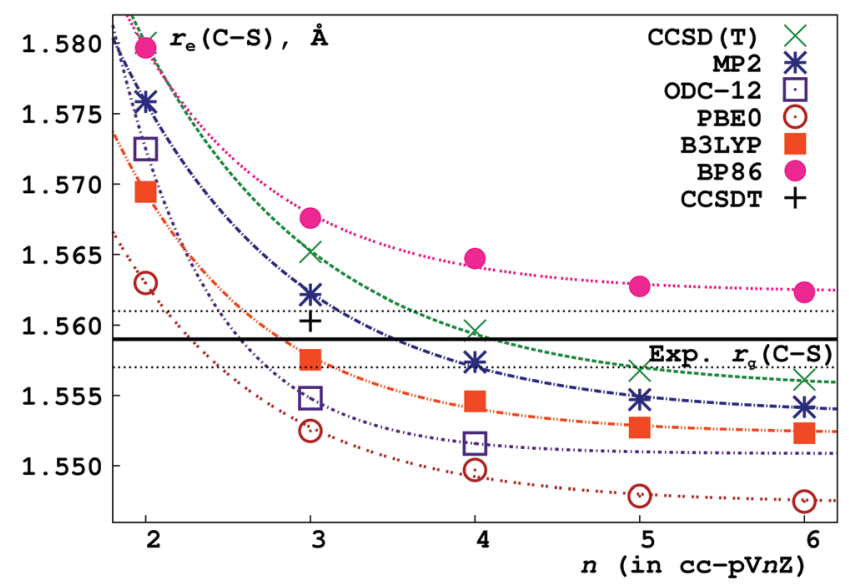

Fig. $3 r_{\mathrm{e}}(\mathrm{C}-\mathrm{S})$ in $\mathrm{CS}_{2}$ at different levels of theory with cc-pVnZ $(n=2, \ldots, 6)$ basis sets. Solid horizontal line represents the experimental $r_{\mathrm{g}}(\mathrm{C}-\mathrm{S})$ bond length $1288 \mathrm{~K}$ and dotted horizontal lines represent its $3 \sigma{ }^{16}$

values of the bond length. Among them only B3LYP is compatible to the post-HF methods mentioned above. Finally ODC-12 (DCFT) shows fast convergence to the value range 1.550-1.552 with cc-pVnZ basis sets, where $n$ is 2 to 4 . BP86 has converged to a value $>r_{\mathrm{g}}(\mathrm{C}-\mathrm{S})$. This illustrates a poor performance of this method for the computation of molecular geometries.

3.1.2 Effects of rotation of the molecule in Monte-Carlo simulations. Let us assume that the classical MC simulation is used to simulate an NVT ensemble. We sample the Boltzmann distribution $\exp \left(-\frac{V}{k T}\right)$, where $V$ is the electronic energy for different arrangements of the nuclei. However, this distribution lacks the rotational degrees of freedom. To account for them, a modified distribution can be used: $\exp \left(-\frac{E}{k T}\right)$, where $E=V+\sum \frac{J_{\alpha}}{2 I_{\alpha}}$, where summation is performed over rotational degrees (their number $N_{\mathrm{r}}=2$ for the linear conformation of 
Table 3 Computed $r_{\mathrm{e}}(\mathrm{C}-\mathrm{S})$ at different levels of theory. All values are in $\AA$

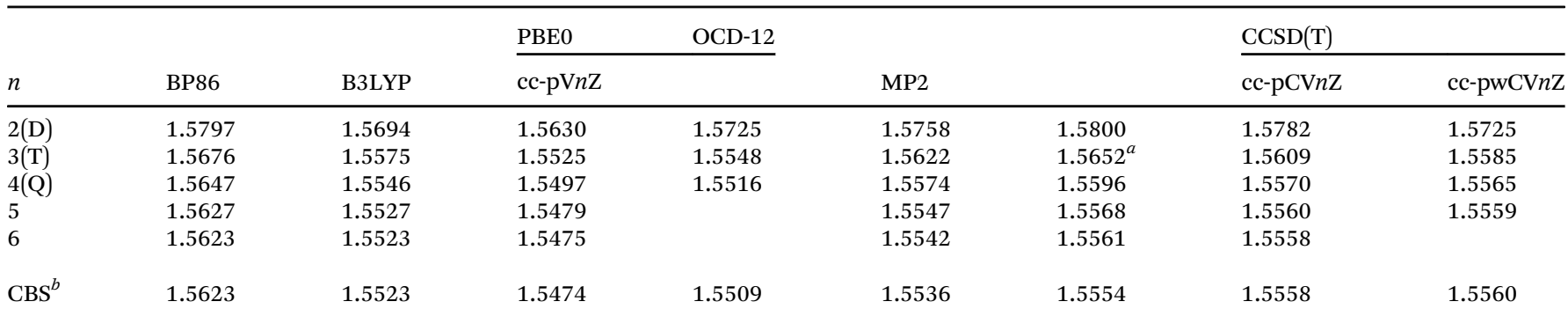

where $n$ is the principle number of the basis set. ${ }^{107} a r_{\mathrm{e}}(\mathrm{C}-\mathrm{S})=1.5603$ A@CCSDT/cc-pVTZ. ${ }^{b}$ Extrapolation to CBS was performed using the formula $r_{\mathrm{e}}(n)=r_{\mathrm{CBS}}+A \exp (-b \cdot n)$.

atoms and $N_{\mathrm{r}}=3$ in the other cases), $I_{\alpha}$ is the main moment of inertia for the molecule. Averaging over the rotational degrees of freedom at certain arrangement of the nuclei leads to the probability distribution $\propto \sqrt{\Pi_{\alpha} I_{\alpha}} \exp \left(-\frac{V}{k T}\right) \cdot{ }^{108}$ Here, the rotation is treated with classical mechanics. In the case of Metropolis Monte-Carlo sampling, each configuration should be weighted using the function $f=\sqrt{\Pi_{\alpha} I_{\alpha}}$. Therefore each observable is computed as $\langle X\rangle=\frac{\langle X \cdot f\rangle}{\langle f\rangle}$, where $X$ is the observed value and $\langle\ldots\rangle$ denotes averaging over the $\mathrm{MC}$ trajectory. In the case of $\mathrm{CS}_{2}$, the $r_{\mathrm{g}}-r_{\mathrm{e}}$ correction for the C-S bond for the MC model without the weighting of the frames with $f$ is $0.12 \mathrm{pm}$, while the value obtained with weighting is $0.17 \mathrm{pm}$.

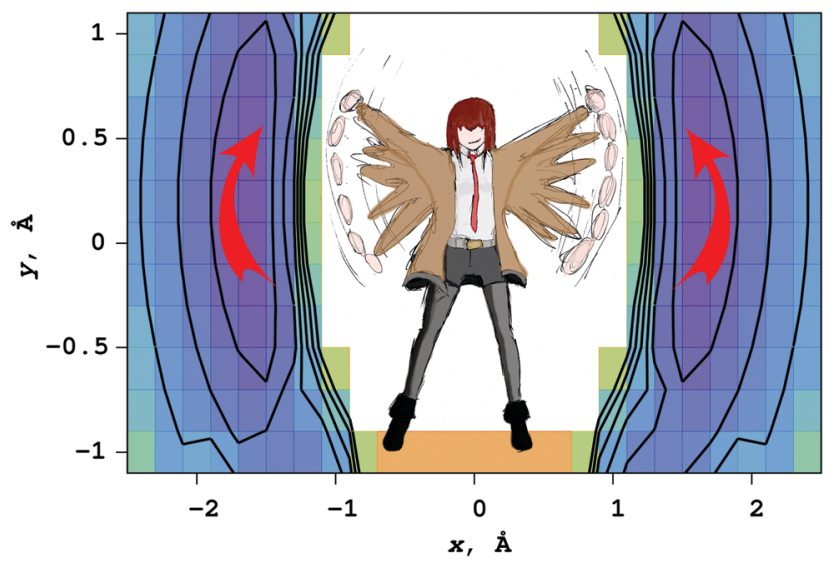

Fig. $4 \mathrm{CS}_{2}$ potential energy surface cross-section at BP86/6-31G level of theory for symmetric stretching and bending vibrations. The abscess axis represents the Cartesian coordinates of the atoms. Carbon atom's coordinates were fixed at $(0,0)$. The coordinates of sulfur atoms were $(x, y)$ and $(-x, y)$.
The difference of $c a .0 .05 \mathrm{pm}$ is of the same order of magnitude as the correction for the rotation of the molecule obtained using VibModule software $(0.03 \mathrm{pm})$. Thus, we find this treatment of rotation in the MC simulations suitable for the rough estimation of the rotational effects.

3.1.3 Shrinkage effect: $r_{\mathrm{e}}$ or $\boldsymbol{r}_{\mathrm{h} \mathbf{1}}$ ? The simplest molecule to observe the anharmonic effects in GED is the $\mathrm{CS}_{2}$. It is the canonical case of the "shrinkage effect". ${ }^{109,110}$ Due to the curvature of the potential, the average distance $-r_{\mathrm{a}}$ or $r_{\mathrm{g}} \mathrm{S} \cdots \mathrm{S}-$ is slightly shorter then the two C-S distances (see Fig. 4). Thus, $\angle$ SCS computed directly from either $r_{\mathrm{a}}$ or $r_{\mathrm{g}}$ will be less than $180^{\circ}$ (see Table 4). ${ }^{111}$ The extension of this effect to polyatomic molecules may lead to misinterpretation of the experimental data and thus false conclusions about the geometrical structure. For instance, the $r_{\mathrm{g}}$ structure of nitrobenzene at $353 \mathrm{~K}$ is not planar: the $\mathrm{NO}_{2}$ group is twisted by approximately $13^{\circ}$ with respect to the benzene ring. ${ }^{17}$ Nevertheless, the structural analysis in terms of $r_{\mathrm{e}}$ structure (i.e. with full accounting of vibrational motions) leads to the planar structure with $C_{2 \mathrm{v}}$ symmetry. ${ }^{18,21}$ Thus, the $r_{\mathrm{g}}$ and $r_{\mathrm{a}}$ structures are geometrically inconsistent, whereas the equilibrium structure $r_{\mathrm{e}}$ is geometrically consistent.

The harmonic approximation results in the $r_{\mathrm{g}}-r_{\alpha}$ corrections ( $r_{\alpha}$ is sometimes denoted $r_{\mathrm{ho}}$ in the GED community). However, the shrinkage effect is not captured by this approximation, since harmonic potential gives a hyperellipsoid-like distribution for the motion of atoms. Some anharmonic effects are introduced by the rotation-vibration interaction, but their order of magnitude is significantly lower that the one of anharmonicity of PES. In order to overcome the problem of inconsistent observable structures without additional computational expenses, a $r_{\mathrm{h} 1}$ structure was proposed. ${ }^{77}$ The idea of this approach is to solve the harmonic vibtational problem in the curvilinear internal coordinates as introduced in ref. Hoy et al. ${ }^{33,112}$

Table 4 Experimental and theoretical thermally averaged parameters of $\mathrm{CS}_{2}$ at $15{ }^{\circ} \mathrm{C}$. Values in parentheses are $3 \sigma .{ }^{16}$ Theoretical $r_{\mathrm{g}}$ values are based on $r_{\mathrm{e}}(\mathrm{C}-\mathrm{S})=1.5560 \AA$, which is a CBS extrapolation for the CCSD(T)/cc-pwCVnZ computational series (see Table 3)

\begin{tabular}{lcccccrrr}
\hline & Exp. $(\mathrm{GED})^{16}$ & ElDiff & GLE + QA & NH + QA & MC + QA & GLE & NH & MC \\
\hline$r_{\mathrm{g}}(\mathrm{C}-\mathrm{S}), \AA$ & $1.559(2)$ & 1.562 & 1.559 & 1.559 & 1.563 & 1.558 & 1.559 \\
$l(\mathrm{C}-\mathrm{S}), \AA$ & $0.036(4)$ & 0.039 & 0.037 & 0.036 & 0.040 & 0.023 & 0.022 \\
$r_{\mathrm{g}}(\mathrm{S} \cdots \mathrm{S}), \AA$ & $3.113(4)$ & 3.116 & 3.114 & 3.127 & 3.114 & 3.114 & 3.113 \\
$l(\mathrm{~S} \cdots \mathrm{S}), \AA$ & $0.045(4)$ & 0.042 & 0.042 & 0.039 & 0.042 & 0.024 & 3.112 \\
$L_{\mathrm{g}}(\mathrm{SCS}),{ }^{\circ}$ & 173 & 172 & 174 & - & 170 & 175 & 174 & 172
\end{tabular}


Table 5 Theoretical $r_{\mathrm{g}}-r_{\mathrm{e}}, r_{\mathrm{g}}-r_{\mathrm{h} 1}$ and $r_{\mathrm{g}}-r_{\alpha}$ corrections for $\mathrm{CS}_{2}$ at $15^{\circ} \mathrm{C}$. All values are in $\mathrm{pm}$

\begin{tabular}{llllll}
\hline & \multicolumn{2}{l}{ Harmonic } & & \\
\cline { 2 - 4 } & Linear $^{b} r_{\mathrm{g}}-r_{\alpha}$ & & \multicolumn{2}{l}{ Curvilinear $r_{\mathrm{g}}-r_{\mathrm{h} 1}$} & \\
\cline { 2 - 4 } Term & ElDiff & & ElDiff & VibModule & \multirow{2}{*}{ Anharmonic ${ }^{a} r_{\mathrm{g}}-r_{\mathrm{e}}$} \\
\hline $\mathrm{C}-\mathrm{S}$ & 0.36 & 0.07 & 0.04 & 0.56 \\
$\mathrm{~S} \cdots \mathrm{S}$ & 0.00 & -0.59 & -0.65 & 0.39
\end{tabular}

${ }^{a}$ The corresponding values were found to be the same for ElDiff with usage of Cartesian, linear and curvilineal internal coordinates as well as for VibModule. ${ }^{b}$ The same values were obtained for Cartesian coordinates using ElDiff.

These coordinates are related to the Cartesian coordinates as $q_{i}=\sum_{k} \frac{\partial q_{i}}{\partial \alpha_{k}} \Delta \alpha_{k}+\frac{1}{2} \sum_{k} \sum_{l} \frac{\partial^{2} q_{i}}{\partial \alpha_{k} \partial \alpha_{l}} \Delta \alpha_{k} \Delta \alpha_{l}$, where $q_{i}$ is the internal curvilinear coordinate, $\alpha_{k}$ is one of the Cartesian coordinates, and $\Delta \alpha_{k}$ is the deviation from the reference value. Vibrational problem is modified by the effective anharmonic term, the so-called "kinematic anharmonicity". It consists of sum of elements $\propto \hat{p}_{i} q_{j} \hat{p}_{k}$, where $\hat{p}_{i}$ is the corresponding momentum of coordinate $q_{i}$. This anharmonic term arises, when the problem is treated in the internal coordinates. After that, perturbation theory is applied to obtain the shrinkage corrections. ${ }^{80}$

The standard implementation of the described algorithm is Shrink software written in Fortran. ${ }^{77-80}$ In this work we use a recent $\mathrm{C}$ implementation in the VibModule program ${ }^{76}$ as well as ElDiff software. ${ }^{15}$ The $\mathrm{CS}_{2}$ molecule was chosen to demonstrate the relation between different methods for calculation of the corrections. The results are presented in Table 5. VibModule uses only the curvilinear internal coordinates. Therefore only two types of corrections were computed with this software. Others were computed with ElDiff.

- $r_{\mathrm{g}}-r_{\alpha}$ (using only the quadratic field).

- $r_{\mathrm{g}}-r_{\mathrm{h} 1}$ (using only the quadratic field).

- $r_{\mathrm{g}}-r_{\mathrm{e}}$ (using the quadratic and cubic force fields), which is equvalent to the VM model.

Obviously, the use of Cartesian coordinates and linearized internal coordinates must result in almost similar results, since the coordinates are connected by a unitary transformation. Nevertheless, they still have been used in a separate fashion yielding the same values for the corrections. The computations using only the harmonic force field in Cartesian and linearized internal coordinates gave $r_{\mathrm{g}}-r_{\alpha}$ corrections, whereas the curvilinear coordinates gave $r_{\mathrm{g}}-r_{\mathrm{h} 1}$ values. The computation of $r_{\mathrm{g}}-r_{\mathrm{e}}$ corrections in all 3 types of coordinates using the quadratic and cubic force fields obviously resulted in the same values, which were in turn equal to the result of the VibModule computation. As we can see, $r_{\mathrm{g}}-r_{\mathrm{e}}, r_{\mathrm{g}}-r_{\mathrm{h} 1}$ and $r_{\mathrm{g}}-r_{\alpha}$ are very different from each other. But if we compute $r_{\mathrm{g}}-r_{\mathrm{e}}$ corrections (or equivalently $r_{\mathrm{a}}-r_{\mathrm{e}}$ ) using other quantum methods, such as PIMD, we will obtain very similar values at the same QC approximation (see ref. Tikhonov et $a l^{54}$ or ESI $\dagger$ for an example). It is due to the clear physical definition of $r_{\mathrm{g}}, r_{\mathrm{a}}$ and $r_{\mathrm{e}}$ values that allows them to be computed. The $r_{\mathrm{h} 1}$ structure is coordinate-dependent and cannot be computed directly. The $r_{\alpha}$ structure is coordinate independent, but is geometrically inconsistent. Also, the $r_{\mathrm{e}}$ structure corresponds to the optimized structure obtained from QC calculations, whether $r_{\mathrm{h} 1}$ and $r_{\alpha}$ cannot be compared with the calculations directly.

Of course, computation of the harmonic force field is significantly cheaper than the cubic one, let alone MD an MC simulations. Still, we assume that it is more convenient to compute the cubic force field or perform MD or MC simulations at a lower QC approximation in order to obtain $r_{\mathrm{a}}-r_{\mathrm{e}}$ corrections in order to perform a direct comparison of the experimental and computed values obtained.

3.1.4 Comparison with the experiment. The results for $\mathrm{CS}_{2}$ are presented in Table 4 . The theoretical $r_{\mathrm{g}}$ distances, amplitudes $l$ and shrinkage angles $L_{\mathrm{g}}$ (SCS) computed from $r_{\mathrm{g}}$ values were compared with the corresponding experimental values. ${ }^{16}$ All the shrinkage angles (both quantum and classical, except for $\mathrm{NH}+\mathrm{QA}$ ) were found to be in good agreement with the experimental one and with each other. The GLE + QA and MC + QA values were slightly smaller then the corresponding classical values as it was expected. It is important to note that the quality of the thermostat plays a very important role for the resulting values of $r_{\mathrm{g}}$ and $r_{\mathrm{a}}$ structures. As we can see the amplitude of S $\cdots \mathrm{S}$ vibrations in the case of $\mathrm{NH}$ was significantly smaller than those observed for GLE and MC. This is can be either a result of the "flying ice cube" effect ${ }^{113}$ (a bad energy redistribution between the degrees of freedom by the thermostat) or the nonergodic trajectory for such few number of degrees of freedom ${ }^{114}$ or their combination. Therefore the extrapolation of quantum effects performed by Qassandra for the $\mathrm{NH}$ model becomes unstable. This leads to unphysical $r_{\mathrm{g}}-r_{\mathrm{e}}$ corrections that make $r_{\mathrm{g}}(\mathrm{S} \cdots \mathrm{S})>2 r_{\mathrm{g}}(\mathrm{C}-\mathrm{S})$.

All the theoretical quantum mean vibrational amplitudes were in significantly better correlation with each other and with experimental data, than those observed for GLE, MC and especially NH. $\mathrm{NH}+$ QA for the $l$-s has performed unexpectedly better than the $r_{\mathrm{g}}-r_{\mathrm{e}}$ corrections at the same level of theory.

\subsection{Four benchmark molecules}

The direct comparison of the terms in the molecules with more then 3 atoms becomes quite boring and inefficient. Therefore similar to the previous work we have performed the comparison of different pure theoretical models for $s M(s)$ with experimental data. The similarity of the theoretical model with experimental data was measured using a structural $R$-factor:

$$
R_{\mathrm{f}}=\sqrt{\frac{\sum_{k=1}^{M}\left(s_{k} M_{\exp }\left(s_{k}\right)-a \cdot s_{k} M_{\text {theor }}\left(s_{k}\right)\right)^{2}}{\sum_{k=1}^{M} s_{k} M_{\exp }^{2}\left(s_{k}\right)}} \cdot 100 \%,
$$

where $a$ is the refined scaling factor. The basic theoretical model in GED is " $r_{\mathrm{e}}$ geometry" + "vibrational parameters $\left(l, r_{\mathrm{a}}-r_{\mathrm{e}}, \kappa\right)$ ". Different optimized structures were obtained using two post-Hartree-Fock methods (in our case - CCSD(T) and MP2) and several popular DFT functionals (B3LYP, PBE0 and BP86) and a very popular cc-pVTZ (and SDB-cc-pVTZ for ICB) basis set. They were used to construct different theoretical 
Table 6 Structural $R_{\mathrm{f}}$ (eqn (8)) for BNZ, CMTC, PZA and ICB with different theoretical geometries and vibrational parameters. cc-pVTZ (and SBD-ccpVTZ for the ICB) basis set was used. All values are in \%

\begin{tabular}{llrrrrr}
\hline Molecule & Vibrational parameters & CCSD(T) & MP2 & PBE0 & B3LYP & BP86 \\
\hline BNZ & ElDiff & 6.8 & 7.2 & 9.6 & 6.8 & 13.2 \\
& PIMD & 8.1 & 7.0 & 13.5 & 9.2 & 9.3 \\
& Q-GLE & 10.0 & 8.9 & 14.6 & 10.8 & 10.4 \\
& GLE + QA & 9.1 & 10.0 & 8.1 & 7.9 & 16.7 \\
& NH + QA & 10.3 & 9.1 & 15.0 & 11.1 & 9.8 \\
& GLE & 19.5 & 19.8 & 19.7 & 19.1 & 23.5 \\
& NH & 22.4 & 21.5 & 26.4 & 23.3 & 20.5 \\
& & & & & & \\
CMTC & VM & 14.2 & 14.4 & 13.7 & 29.0 & 34.0 \\
& NH + QA & 9.8 & 13.1 & 14.9 & 23.2 & 27.0 \\
& NH & 15.5 & 17.4 & 13.8 & 28.3 & 33.0 \\
PZA & ElDiff & & & & & \\
& NH + QA & & 8.9 & 8.2 & 6.5 & 16.1 \\
& NH & & 7.4 & 9.2 & 5.7 & 14.0 \\
& & & 16.2 & 16.5 & 14.9 & 19.7 \\
ICB & ElDiff & & & & & \\
& NH + QA & & 9.2 & 12.2 & 19.4 & 21.3 \\
& NH & & 12.8 & 16.4 & 19.8 & 19.3 \\
& & 19.8 & 23.5 & 24.5 & 23.6
\end{tabular}

models in order to be sure that the observed tendencies in the $R_{\mathrm{f}}$ are not random. The QC approximations for computation of $l, r_{\mathrm{a}}-r_{\mathrm{e}}$, and $\kappa$ were sufficiently less computationally expensive. This is a very common practice in GED that gives quite good results. ${ }^{22-24,54}$

A visualization of the results of GED analysis was done using the so-called radial distribution function $f(r):^{12}$

$$
f(r)=\int_{0}^{\infty} s M(s) \sin (s r) \exp \left(-b \cdot s^{2}\right) \mathrm{d} s,
$$

which is a sine-Fourier transformation of $s M(s)$ with a Gaussian window function $\exp \left(-b \cdot s^{2}\right)$ and $b$ controls the width of the window. The physical meaning of $f(r)$ is the sum of $P(r) / r$ weighted for the scattering ability of the pairs of atoms (see Introduction). The results obtained for $R_{\mathrm{f}} \mathrm{s}$ can be found in Table 6 and the $f(r)$-s for the best available $a b$ initio $r_{\mathrm{e}}$ structure are given in Fig. 5-8.

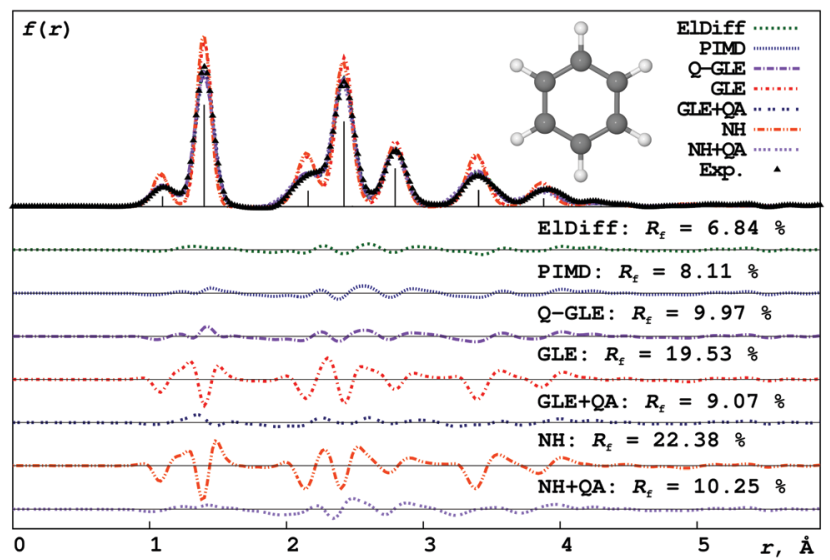

Fig. 5 Experimental (triangles) and theoretical (lines) radial distribution curves and their differences (experimental vs. theoretical) for BNZ. Theoretical models were based on $\operatorname{CCSD}(\mathrm{T}) / \mathrm{cc}-\mathrm{pVTZ}$ equilibrium geometry.

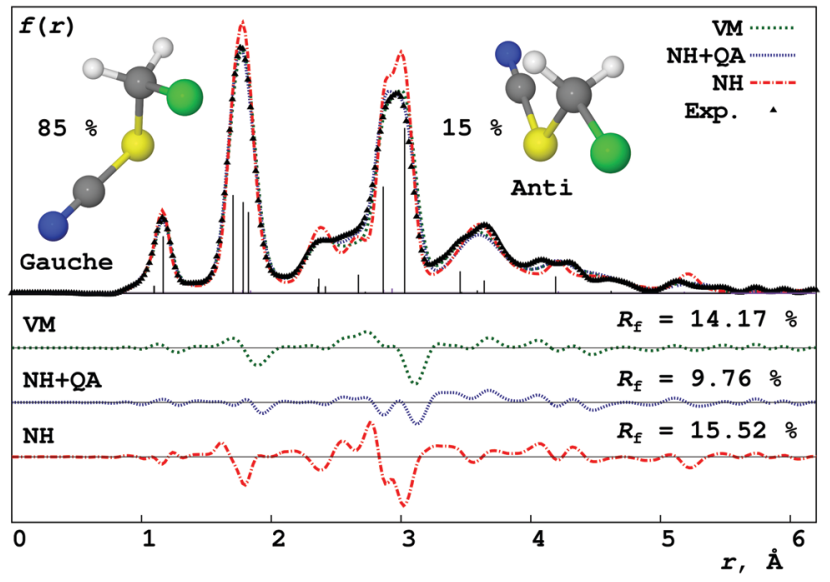

Fig. 6 Experimental (triangles) and theoretical (lines) radial distribution curves and their differences (experimental vs. theoretical) for CMTC. Theoretical models were based on CCSD(T)/cc-pVTZ equilibrium geometry.

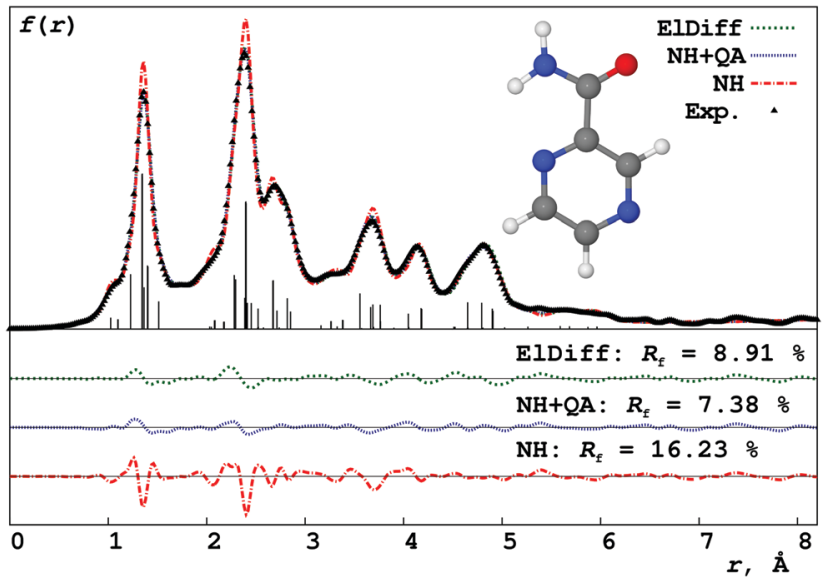

Fig. 7 Experimental (triangles) and theoretical (lines) radial distribution curves and their differences (experimental vs. theoretical) for PZA. Theoretical models were based on MP2/cc-pVTZ equilibrium geometry.

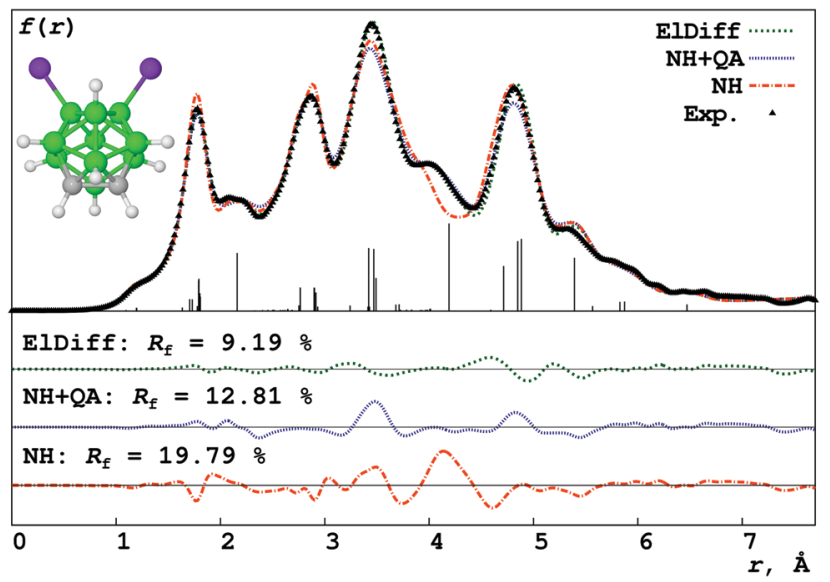

Fig. 8 Experimental (triangles) and theoretical (lines) radial distribution curves and their differences (experimental vs. theoretical) for ICB. Theoretical models were based on MP2/SDB-cc-pVTZ equilibrium geometry. 
3.2.1 Rigid molecules. For the rigid molecules (i.e. BNZ, PZA and ICB) we can see that the tendency is that all the models that account for quantum vibrational effects (ElDiff, PIMD, Q-GLE, $\mathrm{NH}+\mathrm{QA}$ and GLE + QA) give sufficiently better consistency with the experiment than the classical ones (NH and GLE). The influence of quantum effects can be easily seen from $f(r)$ : the peaks from classical models are sufficiently more narrow than from the quantum ones. The ElDiff still gives the best agreement with the experiment in cases of the rigid systems. This is probably the result of the stochastic nature of MD simulations: they give the best result only for the infinite length of the trajectory. Besides, they depend on the performance of the thermostats used. But still the agreement of the MD-based models is satisfactory: they capture most of the vibrational motions' behavior.

We can also see some tendencies for the quality of the predicted geometries from the analysis of Table 6. First of all, MP2 and B3LYP on the chosen set of molecules perform sufficiently well for the molecules that consist of the atoms from the 1st and 2nd rows of the periodic table. But, B3LYP fails in the case of ICB. PBE0, on the other hand, gives quite reasonable geometries for BNZ, PZA and ICB. BP86 is found to give bad geometries. These observations are in good correlation with those for $\mathrm{CS}_{2}$.

3.2.2 Chloromethylthiocyanate: a case of large-amplitude motion. CMTC is a quite unique case in these series of four molecules. First of all it has a LAM: internal rotation of the $\mathrm{CClH}_{2}$ group around the $\mathrm{C}-\mathrm{S}$ bond. This motion brings two possible conformers: gauche and anti (see Fig. 6 and 9). Secondly, CMTC has two atoms from the 3-rd period that are not bonded to each other, whether all other atoms are hydrogens and second period atoms. The $g_{i j}(s)$ 's zeroth approximation for the pair of atoms $i$ and $j$ is $\propto Z_{i} \cdot Z_{j}$, where $Z$ denotes the charge of the atomic nucleus. ${ }^{12}$ Therefore the "visibility" of nonbonded distances is comparable with the bonded distances. This makes the comparison of theoretical models with experimental data very sensible for the conformational effects in the reference $r_{\mathrm{e}}$ geometry.

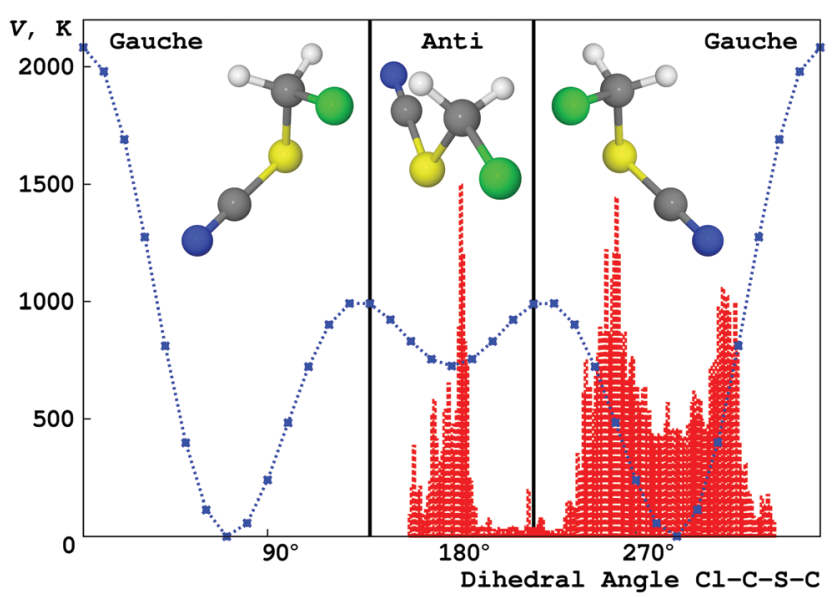

Fig. 9 PES scan (V) and conformational distortion in $\mathrm{NH}$ simulation for CMTC APBE0/def2-SVP.
In order to obtain the vibrational corrections in the conventional model based on quadratic and cubic force fields first ElDiff software was used. However, the calculation yielded imaginary amplitudes for some of the terms due to the small-amplitude motion (SAM) approximation failure. Opposite to ElDiff, VibModule does not use the cubic force field during the computation of $l$-s. Therefore it gives almost the same results in the case of $\mathrm{SAM}^{23,42}$ and still can produce some value in the case of LAM.

The ratio of conformers in all the models was frozen at the theoretical estimation value obtained at the $\operatorname{CCSD}(\mathrm{T}) / \mathrm{cc}-\mathrm{pVTZ}$ level, which was fairly close to the experimental one $(85 \%$ and $89(3) \%$ of gauche at $337 \mathrm{~K}$, respectively). ${ }^{22}$ Treatment of multiple conformers in the MD trajectory is quite simple. The possible values of the dihedral angle $\mathrm{Cl}-\mathrm{C}-\mathrm{S}-\mathrm{C}$ were divided into 3 parts: $\left[0^{\circ} ; 140^{\circ}\right),\left[140^{\circ} ; 220^{\circ}\right)$ and $\left[220^{\circ} ; 360^{\circ}\right)$ (see Fig. 11). The first and the third parts are the gauche conformer, whereas the second one is anti. The computation of this dihedral angle was performed at each step of the trajectory. The frame was assigned to a conformer based on the torsion angle value. This procedure can be easily transferred to the case of multiple LAMs. The division of torsion angles can be performed based on the PES scan (as it was done in the current work) or in the chemical sense. It is due to the assumption that the vicinity of transition states between conformers is not populated. Therefore the small shifts do not matter that much. In other words we can only consider the situations when the conformational distribution has clearly distinguished peaks corresponding to different conformers. If these peaks merge, then the initial cumulant expansion of $s M(s)$ in terms of $l, r_{\mathrm{a}}-r_{\mathrm{e}}$, and $\kappa$ (eqn (3)) does not converge. Therefore a dynamic model should be constructed (examples can be found in ref. Dorofeeva et al., ${ }^{18}$ Khaikin et al., ${ }^{21}$ and Spiridonov et $a .^{25}$ ).

We see that the $\mathrm{NH}+$ QA model with all the initial structures gives the best agreement with the experiment, whether VM is on the same level of agreement with the experiment as NH. We can see from Fig. 8 that the $\mathrm{NH}$ model still gives too narrow peaks than quantum models ( $\mathrm{VM}$ and $\mathrm{NH}+\mathrm{QA})$. The deterioration of the performance of VM is due to the badly approximated $r_{\mathrm{a}}-r_{\mathrm{e}}$ corrections that yield the shifts of the peaks from the experimental positions. This is probably the effect of the SAM model failure. MD-based methods give better sampling of the PES thus yielding a better agreement with the experiment.

Thus we can say that a posteriori corrections introduced in Qassandra can be applied in the case of LAM as well. But only if the conformers are distinguishable. ${ }^{25}$

\subsection{What if we have data from vibrational spectroscopy?}

Unfortunately, in cases of theoretical spectra from MD it is impossible to solve an inverse vibrational problem as opposed to the conventional model based on the quadratic and cubic force fields. Nevertheless it is still possible to perform a direct comparison of the computed spectra with the experimental ones in order to check the quality of the approximation. ${ }^{24}$ It is also fair to note that the most popular method of solving the inverse vibrational problem (i.e. Pulay's method of scaling factors ${ }^{115}$ ) sweeps under the rug all the anharmonic effects. 
It is due to the fact that usually only the harmonic frequencies are fitted to the experimental ones. ${ }^{18,20,21}$ But the anharmonic effect can be very important for the successful assignment of the peaks. ${ }^{36,37}$

The two basic recommendations for the computation of VSp from MD are: ${ }^{99,116}$

- use small time steps.

- obtain several of constant energy MD (i.e. NVE) trajectories under different initial conditions, compute spectra from each of the trajectories and then average them;

The first recommendation reflects the fact that the large time steps shift the vibrational peaks in spectra towards a higher frequency range. ${ }^{4999}$ This behavior, however can be corrected using a correction $\nu_{\text {corr }}=\frac{\sqrt{2 \cdot\left(1-\cos \left(2 \pi \cdot \Delta t \cdot \nu_{\text {ini }}\right)\right)}}{2 \pi \cdot \Delta t}$, where $\nu$ are the initial (ini) and corrected (corr) frequencies and $\Delta t$ is the time step used in the MD simulation. ${ }^{98}$ The only restriction in this case is $\Delta t<1 /\left(\pi \nu_{\max }\right)=\tau_{\min } / \pi$, where $\nu_{\max }$ and $\tau_{\min }$ are the frequency and the period of the fastest motion in the system, respectively. ${ }^{98}$

In cases with GED experimental data presented it is more convenient to perform only one $N V T$ simulation instead. It is due to the fact that $N V E$ trajectories cannot be used for the computation of $l, r_{\mathrm{e}}-r_{\mathrm{a}}$, and $\kappa$. The usage of thermostated NVT MD for the computation of VSp is not novel itself. ${ }^{52,117}$ The aim was to check the performance of the thermostats that give good results for GED as found in this and the previous study ${ }^{54}$ (i.e. NH, GLE and Q-GLE) for generation of theoretical VSp. Experimental IR spectra in the gaseous phase were taken from the NIST Chemistry Webbook. ${ }^{118}$ Theoretical and experimental spectra of $\mathrm{CS}_{2}$, BNZ and PZA can be found in Fig. 10-12.

We can see that $\mathrm{MD}(\mathrm{GLE})$ and $\mathrm{MD}(\mathrm{Q}-\mathrm{GLE})$ spectra are in bad agreement with all the other spectra. Therefore, the corresponding black-box implementations are too noisy to obtain the accurate VSp. $\mathrm{MD}(N V E)$ in the case of BNZ exhibits a poor ratio of the intensities. This is probably the result of the nonequilibrium energy distribution between vibrational degrees of freedom. The NVE trajectory for PZA was thermostatted with chain of two Nose-Hoover thermostats for the initial 1.6 ps of

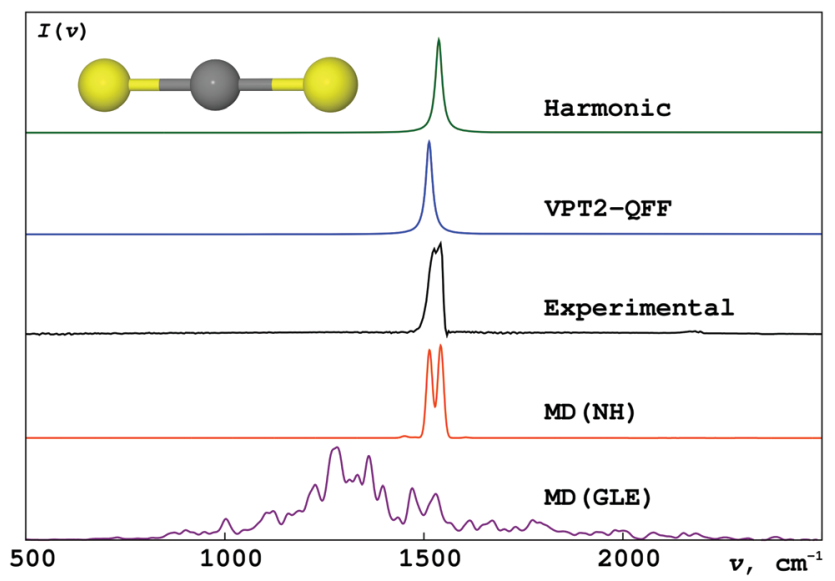

Fig. 10 Experimental and theoretical infrared vibrational spectra of $\mathrm{CS}_{2}$.

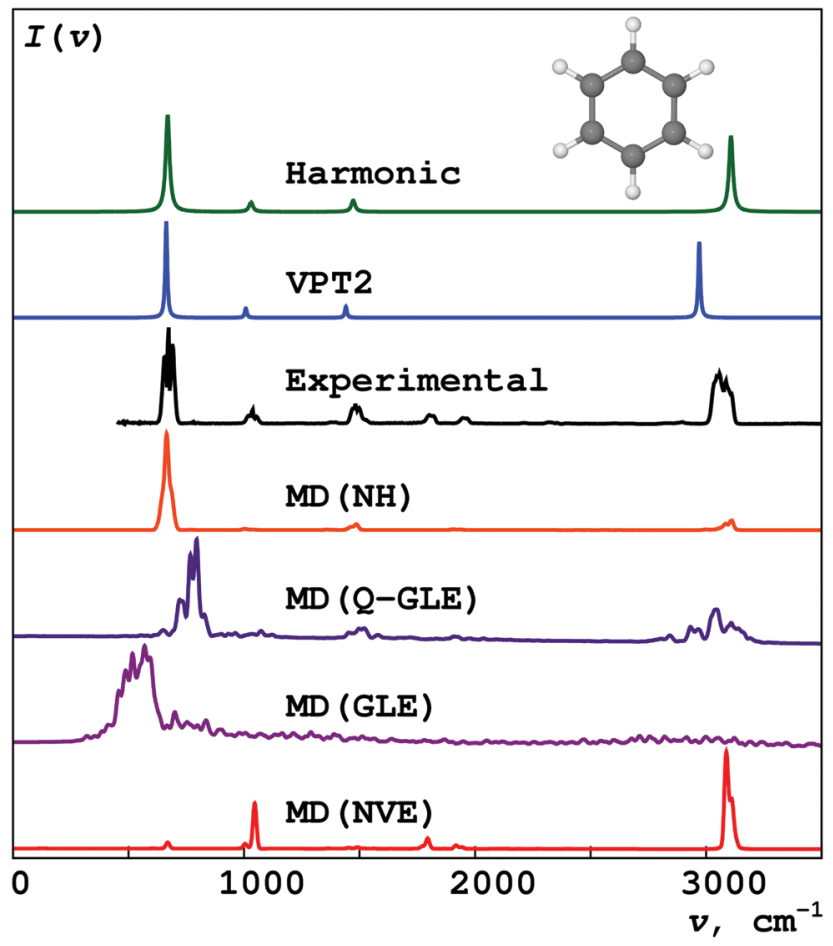

Fig. 11 Experimental and theoretical infrared vibrational spectra of BNZ.

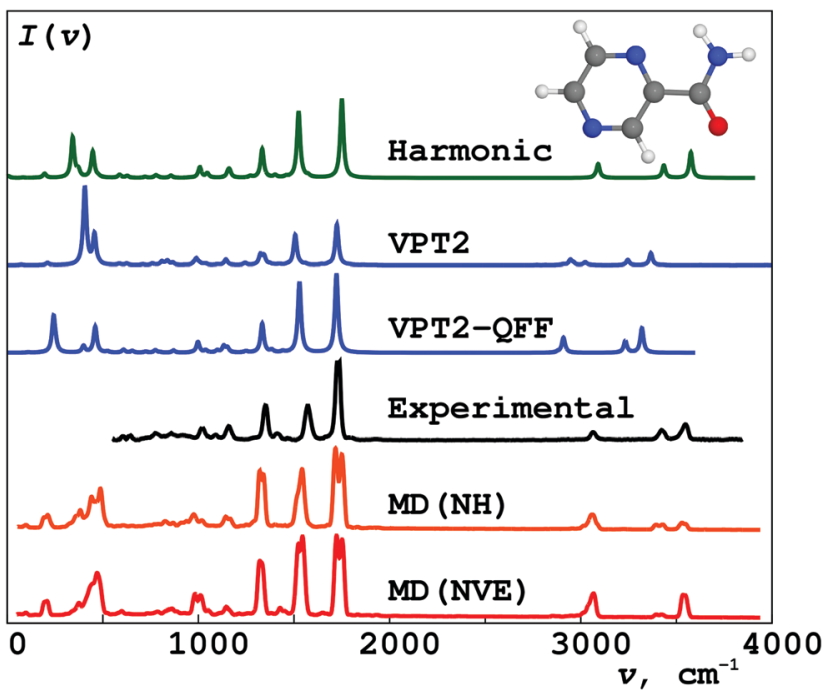

Fig. 12 Experimental and theoretical infrared vibrational spectra of PZA

the trajectory. Therefore the distribution of the intensities in this case is significantly more satisfactory. The NH thermostats show the best agreement with the VPT2 and experimental spectra. However, there is a possibility of significant underestimation of the intensities of high-frequency vibrations. ${ }^{98}$ The reason is a possible energy drain from high-frequency motions to the low frequency ones similar to the "flying ice cube effect". ${ }^{113}$ Thus, in the cases when the experimental VSp are presented we suggest the usage of the Nose-Hoover chain thermostat, but also to be cautious for the possible badly estimated frequencies of the 
fastest vibrations. The colored-noise GLE thermostats parametrized over the solid state unfortunately cannot be used due to the significant contamination of the spectra.

3.3.1 Conclusions and prospectives. The importance of nuclear quantum effects for the correct description of molecular vibrations has been demonstrated previously for the NMR spectra of crystals and in the GED studies of molecules. ${ }^{54119}$ The current work confirms these observations. The applicability of QTB in the form of Q-GLE and a posteriori quantum corrections implemented in Qassandra software was demonstrated once more by comparison with the experimental data for BNZ. Q-GLE, $\mathrm{GLE}+\mathrm{QA}$ and $\mathrm{NH}+\mathrm{QA}$ give very similar results as a more accurate PIMD simulation. A slightly better performance of the conventional model based on the Taylor expansion of the PES can be explained by the fact that the MD trajectories are initiated stochastically (whether or not the integrator itself is stochastic) and are never completely converged. Moreover, quantum effects are introduced only approximately. Thus the MD-based methods are expected to perform better than the conventional ones for larger non-rigid molecules, where the loworder Taylor expansion fails to represent the PES.

The comparison of the five test objects has revealed an acceptable performance of a posteriori quantum corrections (NH + QA and GLE + QA models). This correction was shown to be applicable to the classical $\mathrm{MC}$ simulations using $\mathrm{CS}_{2}$ as an example. The initial MD sampling quality was found to be very important: $\mathrm{NH}+\mathrm{QA}$ failed to describe correctly the shrinkage effect of $\mathrm{CS}_{2}$, while $\mathrm{MC}+\mathrm{QA}$ and GLE + QA demonstrated rather stable results. Importantly, this was not observed for the rigid aromatic molecules studied in our previous work, ${ }^{54}$ where quantumcorrected NH-trajectories exhibited equally good accuracy.

The applicability of the quantum corrections for the systems with large-amplitude motion has been demonstrated for the first time using CMTC as a test object. The idea of treatment of MD or MC results is the following: the trajectory should be divided into parts that are assigned to different conformers, the vibrational parameters $l, r_{\mathrm{e}}-r_{\mathrm{a}}$ and $\kappa$ being computed for each of them similarly to the small-amplitude motion case. This algorithm is only applicable if the conformational distribution has the resolved regions corresponding to different conformers. In other words, the probability of the molecule to be "between" the conformers should be negligible. Fortunately, this is also the basic assumption beyond the GED model based on eqn (3) with parameters $l, r_{\mathrm{e}}-r_{\mathrm{a}}$ and $\kappa$. For the systems with significantly larger-amplitude vibrations, the direct treatment of these motion types should be performed (for example via a dynamic model).

It would be tempting to extract all the required information for GED, VSp or RSp together from one MD or MC trajectory. However, this is currently not possible due to several limitations (see the summary in Fig. 13.). First, MC simulations are currently unable to reproduce VSp in the whole range of frequencies. Therefore, if GED and VSp data are given they can be analyzed together only through MD simualtions. The situtation is significantly worse for RSp: classical simulations are by definition inapplicable, since the observables in the experiments are the rotational constants $(B)$ that correspond to some vibrational level.

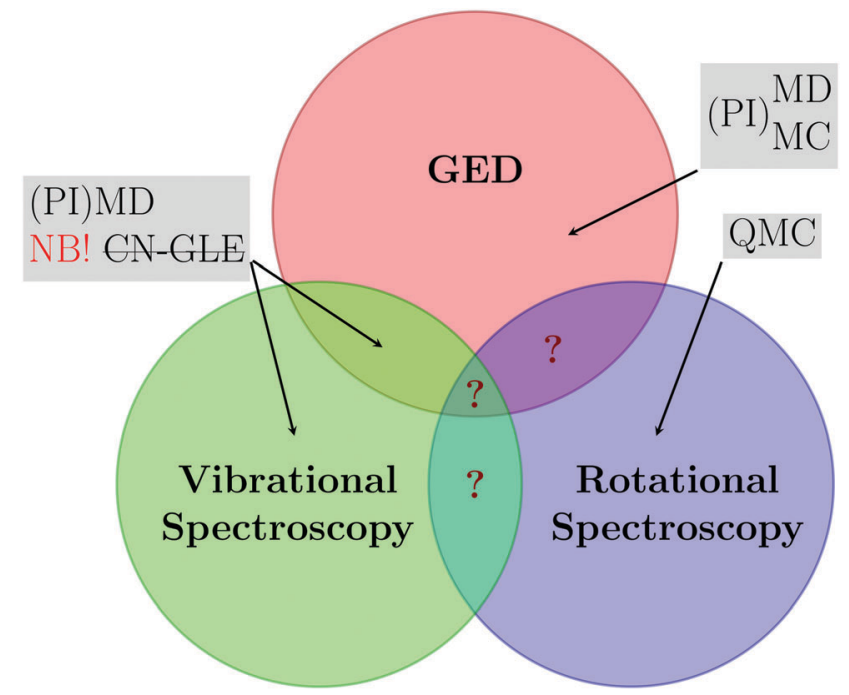

Fig. 13 Applicability of different stochastic simulations for GED, VSp and RSp.

Usually it is the zeroth level and the constants are consequently denoted as $B_{0}$. Stochastic simulations in this case are still applicable. For example, in the work of Liu et al. ${ }^{120}$ the arrangement of the water molecules in the hexamer was determined using quantum diffusion $\mathrm{MC}^{121}$ calculations of $B_{0}$. Nevertheless, classical simulations have been proven to be useful tools for the prediction and interpretation of the molecular structure determining experiments that are both computationally feasible and sufficiently accurate.

\section{Acknowledgements}

This work was supported by Deutsche Forschungsgemeinschaft (DFG, projects VI 713/1-1 and CRC953) and NCCR MARVEL funded by the Swiss National Science Foundation. Calculations performed by V. R. were enabled by the Swiss National Supercomputer Centre (CSCS) under Project ID ch5. D. S. would like to thank Regionales Rechenzentrum Erlangen for the computational time. D. T. is grateful for the computational time and programs offered by the Supercomputing Center of Lomonosov Moscow State University ${ }^{122}$ and computational resources of Laboratory of Inorganic and Structural Chemistry (University of Bielefeld). The authors would like to express their gratitude to $\mathrm{Dr} \mathrm{Yu}$. V. Vishnevskiy for initial GED data processing for BNZ, for performing VPT2 computations for PZA and also for providing computational and experimental data for CMTC.

\section{References}

1 L. Pauling and L. O. Brockway, J. Chem. Phys., 1934, 2, 867-881. 2 L. O. Brockway, Rev. Mod. Phys., 1936, 8, 231-266.

3 A. J. Stosick, J. Am. Chem. Soc., 1939, 61, 1127-1130.

4 G. Herzberg, Infrared and Raman Spectra of Polyatomic Molecules, Krieger Publishing Company, 1945.

5 L. Pauling, The Nature of the Chemical Bond and the Structure of Molecules and Crystals: An Introduction to Modern Structural Chemistry, Cornell University Press, 1960. 
6 R. J. Gillespie and I. Hargittai, The VSEPR Model of Molecular Geometry, Dover Publications, Incorporated, 2012.

7 I. Hargittai, in Gas-Phase Electron Diffraction for Molecular Structure Determination, ed. T. E. Weirich, J. L. Lábár and X. Zou, Springer, Netherlands, Dordrecht, 2006, pp. 197-206.

8 M. J. S. Dewar and W. Thiel, J. Am. Chem. Soc., 1977, 99, 4899-4907.

9 M. J. S. Dewar and W. Thiel, J. Am. Chem. Soc., 1977, 99, 4907-4917.

10 M. J. S. Dewar, E. G. Zoebisch, E. F. Healy and J. J. P. Stewart, J. Am. Chem. Soc., 1985, 107, 3902-3909.

11 H. S. Yu, X. He and D. G. Truhlar, J. Chem. Theory Comput., 2016, 12, 1280-1293.

12 I. Hargittai, in Stereochemical Applications of Gas Phase Electron Diffraction, Part A: The Electron Diffraction Technique, ed. I. Hargittai and M. Hargittai, VCH Publishers, Inc., New York, 1988.

13 R. S. Drago, Physical methods for chemists, Saunders College Pub., 1992.

14 A. G. Yagola, I. V. Kochikov, G. M. Kuramshina and Y. A. Pentin, Inverse problems of vibrational spectroscopy, VSP BV, 1999.

15 I. V. Kochikov, Y. I. Tarasov, G. M. Kuramshina, V. P. Spiridonov, A. G. Yagola and T. G. Strand, J. Mol. Struct., 1998, 445, 243-258.

16 Y. Morino and T. Iijima, Bull. Chem. Soc. Jpn., 1962, 35, 1661-1667.

17 A. Domenicano, G. Schultz, I. Hargittai, M. Colapietro, G. Portalone, P. George and C. W. Bock, Struct. Chem., 1990, 1, 107-122.

18 O. V. Dorofeeva, Y. Vishnevskiy, N. Vogt, J. Vogt, L. Khristenko, S. Krasnoshchekov, I. Shishkov, I. Hargittai and L. Vilkov, Struct. Chem., 2007, 18, 739-753.

19 Y. I. Tarasov, I. V. Kochikov, D. M. Kovtun, N. Vogt, B. K. Novosadov and A. S. Saakyan, J. Struct. Chem., 2004, 45, 778-785.

20 N. Vogt, L. S. Khaikin, O. E. Grikina and A. N. Rykov, J. Mol. Struct., 2013, 1050, 114-121.

21 L. S. Khaikin, I. V. Kochikov, O. E. Grikina, D. S. Tikhonov and E. G. Baskir, Struct. Chem., 2015, 26, 1651-1687.

22 Y. Berrueta Martinez, L. S. Rodriguez Pirani, M. F. Erben, C. G. Reuter, Y. V. Vishnevskiy, H. G. Stammler, N. W. Mitzel and C. O. Della Vedova, Phys. Chem. Chem. Phys., 2015, 17, 15805-15812.

23 Y. V. Vishnevskiy, D. S. Tikhonov, C. G. Reuter, N. W. Mitzel, D. Hnyk, J. Holub, D. A. Wann, P. D. Lane, R. J. F. Berger and S. A. Hayes, Inorg. Chem., 2015, 54, 11868-11874.

24 D. S. Tikhonov, Y. V. Vishnevskiy, A. N. Rykov, O. E. Grikina and L. S. Khaikin, J. Mol. Struct., 2016, DOI: 10.1016/ j.molstruc.2016.05.090.

25 V. P. Spiridonov, A. A. Ishchenko and E. Z. Zasorin, Russ. Chem. Rev., 1978, 47, 54.

26 N. Vogt, J. Mol. Struct., 2001, 570, 189-195.

27 F. Pawłowski, P. Jørgensen, J. Olsen, F. Hegelund, T. Helgaker, J. Gauss, K. L. Bak and J. F. Stanton, J. Chem. Phys., 2002, 116, 6482-6496.
28 D. G. Artiukhin, J. Kłos, E. J. Bieske and A. A. Buchachenko, J. Phys. Chem. A, 2014, 118, 6711-6720.

29 L. A. Surin, A. Potapov, A. A. Dolgov, I. V. Tarabukin, V. A. Panfilov, S. Schlemmer, Y. N. Kalugina, A. Faure and A. van der Avoird, J. Chem. Phys., 2015, 142, 114308.

30 L. A. Surin, I. V. Tarabukin, V. A. Panfilov, S. Schlemmer, Y. N. Kalugina, A. Faure, C. Rist and A. van der Avoird, J. Chem. Phys., 2015, 143, 154303.

31 J. C. D. E. Bright Wilson, Jr., Molecular Vibrations: The Theory of Infrared and Raman Vibrational Spectra, Dover Publications, 1980.

32 P. Atkins and J. De Paula, Physical Chemistry, Oxford University Press, 8th edn, 2006.

33 P. R. Bunker and P. Jensen, Molecular Symmetry and Spectroscopy, NRC Research Press, 2nd edn, 2016, p. 748.

34 I. V. Kochikov, Y. I. Tarasov, N. Vogt and V. P. Spiridonov, J. Mol. Struct., 2002, 607, 163-174.

35 C. Puzzarini and V. Barone, Phys. Chem. Chem. Phys., 2011, 13, 7189-7197.

36 S. V. Krasnoshchekov and N. F. Stepanov, J. Phys. Chem. A, 2015, 119, 1616-1627.

37 S. V. Krasnoshchekov, N. Vogt and N. F. Stepanov, J. Phys. Chem. A, 2015, 119, 6723-6737.

38 V. Barone, J. Chem. Phys., 2005, 122, 014108.

39 F. Jensen, Introduction to Computational Chemistry, John Wiley \& Sons, Inc., 1999.

40 D. Young, Computational Chemistry: A Practical Guide for Applying Techniques to Real World Problems, John Wiley \& Sons, Inc., 2001.

41 T. Schlick, Molecular Modeling and Simulation: An Interdisciplinary Guide, Springer, 2010.

42 Y. V. Vishnevskiy and D. Tikhonov, Theor. Chem. Acc., 2016, 135, 88.

43 Y. A. Zhabanov, A. V. Zakharov, S. A. Shlykov, O. N. Trukhina, E. A. Danilova, O. I. Koifman and M. K. Islyaikin, J. Porphyrins Phthalocyanines, 2013, 17, 220-228.

44 O. A. Pimenov, N. I. Giricheva, S. Blomeyer, V. E. Mayzlish, N. W. Mitzel and G. V. Girichev, Struct. Chem., 2015, 26, 1531-1541.

45 Y. A. Zhabanov, A. V. Zakharov, N. I. Giricheva, S. A. Shlykov, O. I. Koifman and G. V. Girichev, J. Mol. Struct., 2015, 1092, 104-112.

46 J. S. Tse, Annu. Rev. Phys. Chem., 2002, 53, 249-290.

47 W. B. Bosma, L. E. Fried and S. Mukamel, J. Chem. Phys., 1993, 98, 4413-4421.

48 S. Iuchi, A. Morita and S. Kato, J. Phys. Chem. B, 2002, 106, 3466-3476.

49 M. Praprotnik and D. Janežič, J. Chem. Phys., 2005, 122, 174103.

50 S. D. Ivanov, A. Witt and D. Marx, Phys. Chem. Chem. Phys., 2013, 15, 10270-10299.

51 R. Ramírez, T. López-Ciudad, P. Kumar and D. Marx, J. Chem. Phys., 2004, 121, 3973-3983.

52 D. A. Wann, A. V. Zakharov, A. M. Reilly, P. D. McCaffrey and D. W. H. Rankin, J. Phys. Chem. A, 2009, 113, 9511-9520. 
53 K. Kuchitsu, Bull. Chem. Soc. Jpn., 1967, 40, 498-504.

54 D. S. Tikhonov, A. Otlyotov and V. Rybkin, Phys. Chem. Chem. Phys., 2016, 18, 18237-18245.

55 M. J. Frisch, G. W. Trucks, H. B. Schlegel, G. E. Scuseria, M. A. Robb, J. R. Cheeseman, G. Scalmani, V. Barone, B. Mennucci, G. A. Petersson, H. Nakatsuji, M. Caricato, X. Li, H. P. Hratchian, A. F. Izmaylov, J. Bloino, G. Zheng, J. L. Sonnenberg, M. Hada, M. Ehara, K. Toyota, R. Fukuda, J. Hasegawa, M. Ishida, T. Nakajima, Y. Honda, O. Kitao, H. Nakai, T. Vreven, J. A. Montgomery, J. E. Peralta, F. Ogliaro, M. Bearpark, J. J. Heyd, E. Brothers, K. N. Kudin, V. N. Staroverov, R. Kobayashi, J. Normand, K. Raghavachari, A. Rendell, J. C. Burant, S. S. Iyengar, J. Tomasi, M. Cossi, N. Rega, J. M. Millam, M. Klene, J. E. Knox, J. B. Cross, V. Bakken, C. Adamo, J. Jaramillo, R. Gomperts, R. E. Stratmann, O. Yazyev, A. J. Austin, R. Cammi, C. Pomelli, J. W. Ochterski, R. L. Martin, K. Morokuma, V. G. Zakrzewski, G. A. Voth, P. Salvador, J. J. Dannenberg, S. Dapprich, A. D. Daniels, O. Farkas, J. B. Foresman, J. V. Ortiz, J. Cioslowski and D. J. Fox, Gaussian 09, Revision D.01, Gaussian Inc., Wallingford, CT, 2009.

56 J. M. Turney, A. C. Simmonett, R. M. Parrish, E. G. Hohenstein, F. A. Evangelista, J. T. Fermann, B. J. Mintz, L. A. Burns, J. J. Wilke, M. L. Abrams, N. J. Russ, M. L. Leininger, C. L. Janssen, E. T. Seidl, W. D. Allen, H. F. Schaefer, R. A. King, E. F. Valeev, C. D. Sherrill and T. D. Crawford, Wiley Interdiscip. Rev.: Comput. Mol. Sci., 2012, 2, 556-565.

57 CFOUR, a quantum chemical program package written by J. F. Stanton, J. Gauss, M. E. Harding, P. G. Szalay with contributions from A. A. Auer, R. J. Bartlett, U. Benedikt, C. Berger, D. E. Bernholdt, Y. J. Bomble, L. Cheng, O. Christiansen, M. Heckert, O. Heun, C. Huber, T.-C. Jagau, D. Jonsson, J. Jusélius, K. Klein, W. J. Lauderdale, D. A. Matthews, T. Metzroth, L. A. Mück, D. P. O’Neill, D. R. Price, E. Prochnow, C. Puzzarini, K. Ruud, F. Schiffmann, W. Schwalbach, C. Simmons, S. Stopkowicz, A. Tajti, J. Vázquez, F. Wang, J. D. Watts and the integral packages MOLECULE (J. Almlöf and P. R. Taylor), PROPS (P. R. Taylor), ABACUS (T. Helgaker, H. J. Aa. Jensen, P. Jørgensen, and J. Olsen), and ECP routines by A. V. Mitin and C. van Wüllen. For the current version, see http://www.cfour.de., 2016.

58 A. A. Granovsky, Firefly version 8 , http://classic.chem.msu. su/gran/firefly/index.html.

59 S. Gundersen, S. Samdal, T. G. Strand and H. V. Volden, J. Mol. Struct., 2007, 832, 164-171.

60 T. D. Crawford and H. F. Schaefer, An Introduction to Coupled Cluster Theory for Computational Chemists, John Wiley \& Sons, Inc., 2007, pp. 33-136.

61 C. Møller and M. S. Plesset, Phys. Rev., 1934, 46, 618-622.

62 A. Y. Sokolov and H. F. Schaefer, J. Chem. Phys., 2013, 139, 204110.

63 C. Adamo and V. Barone, J. Chem. Phys., 1999, 110, 6158-6170.
64 A. D. Becke, J. Chem. Phys., 1993, 98, 5648-5652.

65 C. Lee, W. Yang and R. G. Parr, Phys. Rev. B: Condens. Matter Mater. Phys., 1988, 37, 785-789.

66 S. H. Vosko, L. Wilk and M. Nusair, Can. J. Phys., 1980, 58, 1200-1211.

67 A. D. Becke, Phys. Rev. A: At., Mol., Opt. Phys., 1988, 38, 3098-3100.

68 J. P. Perdew, Phys. Rev. B: Condens. Matter Mater. Phys., 1986, 33, 8822-8824.

69 J. P. Perdew and A. Zunger, Phys. Rev. B: Condens. Matter Mater. Phys., 1981, 23, 5048-5079.

70 T. H. Dunning, J. Chem. Phys., 1989, 90, 1007-1023.

71 D. E. Woon and T. H. Dunning, J. Chem. Phys., 1995, 103, 4572-4585.

72 K. A. Peterson and T. H. Dunning, J. Chem. Phys., 2002, 117, 10548-10560.

73 J. M. L. Martin and A. Sundermann, J. Chem. Phys., 2001, 114, 3408-3420.

74 K. L. Schuchardt, B. T. Didier, T. Elsethagen, L. Sun, V. Gurumoorthi, J. Chase, J. Li and T. L. Windus, J. Chem. Inf. Model., 2007, 47, 1045-1052.

75 D. Feller, J. Comput. Chem., 1996, 17, 1571-1586.

76 Y. V. Vishnevskiy and Y. A. Zhabanov, J. Phys.: Conf. Ser., 2015, 633, 012076.

77 V. A. Sipachev, J. Mol. Struct.: THEOCHEM, 1985, 121, 143-151.

78 V. A. Sipachev, Struct. Chem., 2000, 11, 167-172.

79 V. A. Sipachev, J. Mol. Struct., 2001, 567-568, 67-72.

80 V. A. Sipachev, Adv. Phys. Chem., 2011, 2011, 1-14.

81 S. Nosé, J. Chem. Phys., 1984, 81, 511-519.

82 W. G. Hoover, Phys. Rev. A: At., Mol., Opt. Phys., 1985, 31, 1695-1697.

83 G. J. Martyna, M. L. Klein and M. Tuckerman, J. Chem. Phys., 1992, 97, 2635-2643.

84 M. Ceriotti, G. Bussi and M. Parrinello, Phys. Rev. Lett., 2009, 102, 020601.

85 N. Metropolis, A. W. Rosenbluth, M. N. Rosenbluth, A. H. Teller and E. Teller, J. Chem. Phys., 1953, 21, 1087-1092.

86 M. Ceriotti, G. Bussi and M. Parrinello, Phys. Rev. Lett., 2009, 103, 030603.

87 M. Ceriotti, G. Bussi and M. Parrinello, J. Chem. Theory Comput., 2010, 6, 1170-1180.

88 M. W. Schmidt, K. K. Baldridge, J. A. Boatz, S. T. Elbert, M. S. Gordon, J. H. Jensen, S. Koseki, N. Matsunaga, K. A. Nguyen, S. Su, T. L. Windus, M. Dupuis and J. A. Montgomery, J. Comput. Chem., 1993, 14, 1347-1363.

89 The CP2K developers group, $\mathrm{CP} 2 \mathrm{~K}$ is freely available from: http://www.cp2k.org/, 2014.

90 J. VandeVondele, M. Krack, F. Mohamed, M. Parrinello, T. Chassaing and J. Hutter, Comput. Phys. Commun., 2005, 167, 103-128.

91 J. Hutter, M. Iannuzzi, F. Schiffmann and J. VandeVondele, Wiley Interdiscip. Rev.: Comput. Mol. Sci., 2014, 4, 15-25.

92 M. J. Frisch, G. W. Trucks, H. B. Schlegel, G. E. Scuseria, M. A. Robb, J. R. Cheeseman, J. A. Montgomery, Jr., T. Vreven, K. N. Kudin, J. C. Burant, J. M. Millam, S. S. Iyengar, 
J. Tomasi, V. Barone, B. Mennucci, M. Cossi, G. Scalmani, N. Rega, G. A. Petersson, H. Nakatsuji, M. Hada, M. Ehara, K. Toyota, R. Fukuda, J. Hasegawa, M. Ishida, T. Nakajima, Y. Honda, O. Kitao, H. Nakai, M. Klene, X. Li, J. E. Knox, H. P. Hratchian, J. B. Cross, V. Bakken, C. Adamo, J. Jaramillo, R. Gomperts, R. E. Stratmann, O. Yazyev, A. J. Austin, R. Cammi, C. Pomelli, J. W. Ochterski, P. Y. Ayala, K. Morokuma, G. A. Voth, P. Salvador, J. J. Dannenberg, V. G. Zakrzewski, S. Dapprich, A. D. Daniels, M. C. Strain, O. Farkas, D. K. Malick, A. D. Rabuck, K. Raghavachari, J. B. Foresman, J. V. Ortiz, Q. Cui, A. G. Baboul, S. Clifford, J. Cioslowski, B. B. Stefanov, G. Liu, A. Liashenko, P. Piskorz, I. Komaromi, R. L. Martin, D. J. Fox, T. Keith, M. A. Al-Laham, C. Y. Peng, A. Nanayakkara, M. Challacombe, P. M. W. Gill, B. Johnson, W. Chen, M. W. Wong, C. Gonzalez and J. A. Pople, Gaussian 03, Revision D.02, Gaussian, Inc., Wallingford, CT, 2004.

93 J. VandeVondele and J. Hutter, J. Chem. Phys., 2007, 127, 114105.

94 K. Yagi, K. Hirao, T. Taketsugu, M. W. Schmidt and M. S. Gordon, J. Chem. Phys., 2004, 121, 1383-1389.

95 S. Goedecker, M. Teter and J. Hutter, Phys. Rev. B: Condens. Matter Mater. Phys., 1996, 54, 1703-1710.

96 G. Lippert, J. Hutter and M. Parrinello, Mol. Phys., 1997, 92, 477-488.

97 J. Kolafa, J. Comput. Chem., 2004, 25, 335-342.

98 D. S. Tikhonov, J. Chem. Phys., 2016, 144, 174108.

99 J. Horníček, P. Kaprálová and P. Bouř, J. Chem. Phys., 2007, 127, 084502.

100 A.-R. Allouche, J. Comput. Chem., 2011, 32, 174-182.

101 Y. V. Vishnevskiy, UNEX version 1.6, http://unexprog.org, 2015.

102 R. J. F. Berger, M. Hoffmann, S. A. Hayes and N. W. Mitzel, Z. Naturforsch., B: J. Chem. Sci., 2009, 64b, 1259-1268.

103 C. G. Reuter, Y. V. Vishnevskiy, S. Blomeyer and N. W. Mitzel, Z. Naturforsch., B: J. Chem. Sci., 2016, 71, 1-13.
104 Y. V. Vishnevskiy, J. Mol. Struct., 2007, 833, 30-41.

105 Y. V. Vishnevskiy, J. Mol. Struct., 2007, 871, 24-32.

106 A. Halkier, P. Jørgensen, J. Gauss and T. Helgaker, Chem. Phys. Lett., 1997, 274, 235-241.

107 B. Paizs, P. Salvador, A. G. Császár, M. Duran and S. Suhai, J. Comput. Chem., 2001, 22, 196-207.

108 L. Landau and E. Lifshits, Statistical Physics. Part I., Heinemann, Butterworth, 3rd edn, 1999, vol. 5.

109 O. Bastiansen and M. Trætteberg, Acta Crystallogr., 1960, 13, 1108.

110 Y. Morino, Acta Crystallogr., 1960, 13, 1107.

111 Y. Morino, J. Nakamura and P. W. Moore, J. Chem. Phys., 1962, 36, 1050-1056.

112 A. R. Hoy, I. M. Mills and G. Strey, Mol. Phys., 1972, 24, 1265-1290.

113 S. C. Harvey, R. K.-Z. Tan and T. E. Cheatham, J. Comput. Chem., 1998, 19, 726-740.

114 P. K. Patra and B. Bhattacharya, Phys. Rev. E: Stat., Nonlinear, Soft Matter Phys., 2014, 90, 043304.

115 P. Pulay, G. Fogarasi, G. Pongor, J. E. Boggs and A. Vargha, J. Am. Chem. Soc., 1983, 105, 7037-7047.

116 S. A. Fischer, T. W. Ueltschi, P. Z. El-Khoury, A. L. Mifflin, W. P. Hess, H.-F. Wang, C. J. Cramer and N. Govind, J. Phys. Chem. B, 2016, 120, 1429-1436.

117 V. Agarwal, G. W. Huber, W. C. Conner and S. M. Auerbach, J. Chem. Phys., 2011, 135, 134506.

118 NIST Chemistry WebBook, http://webbook.nist.gov.

119 M. Dračínský, P. Bouř and P. Hodgkinson, J. Chem. Theory Comput., 2016, 12, 968-973.

120 K. Liu, M. G. Brown, C. Carter, R. J. Saykally, J. K. Gregory and D. C. Clary, Nature, 1996, 381, 501-503.

121 W. M. C. Foulkes, L. Mitas, R. J. Needs and G. Rajagopal, Rev. Mod. Phys., 2001, 73, 33-83.

122 V. V. Voevodin, S. A. Zhumatiy, S. I. Sobolev, A. S. Antonov, P. A. Bryzgalov, D. A. Nikitenko, K. S. Stefanov and V. V. Voevodin, Open Syst. J., 2012, 36-39. 